\title{
Comparison between vortices created and evolving during fixed and dynamic solar wind conditions
}

\author{
Y. M. Collado-Vega ${ }^{1}$, R. L. Kessel ${ }^{2}$, D. G. Sibeck ${ }^{1}$, V. L. Kalb ${ }^{3}$, R. A. Boller ${ }^{4}$, and L. Rastaetter ${ }^{1}$ \\ ${ }^{1}$ NASA Goddard Space Flight Center, Space Weather Laboratory, Code 674, Greenbelt, MD, USA \\ ${ }^{2}$ NASA Headquarters, SMD, Heliophysics Division, Washington, D.C., USA \\ ${ }^{3}$ NASA Goddard Space Flight Center, Terrestrial Information Systems Laboratory, Code 619, Greenbelt, MD, USA \\ ${ }^{4}$ NASA Goddard Space Flight Center, Science Data Systems Branch, Code 586, Greenbelt, MD, USA \\ Correspondence to: Y. M. Collado-Vega (yaireska.m.colladovega@ nasa.gov)
}

Received: 12 January 2013 - Revised: 28 June 2013 - Accepted: 1 July 2013 - Published: 30 August 2013

\begin{abstract}
We employ Magnetohydrodynamic (MHD) simulations to examine the creation and evolution of plasma vortices within the Earth's magnetosphere for steady solar wind plasma conditions. Very few vortices form during intervals of such solar wind conditions. Those that do remain in fixed positions for long periods (often hours) and exhibit rotation axes that point primarily in the $x$ or $y$ direction, parallel (or antiparallel) to the local magnetospheric magnetic field direction. Occasionally, the orientation of the axes rotates from the $x$ direction to another direction. We compare our results with simulations previously done for unsteady solar wind conditions. By contrast, these vortices that form during intervals of varying solar wind conditions exhibit durations ranging from seconds (in the case of those with axes in the $x$ or $y$ direction) to minutes (in the case of those with axes in the $z$ direction) and convect antisunward. The local-time dependent sense of rotation seen in these previously reported vortices suggests an interpretation in terms of the Kelvin-Helmholtz instability. For steady conditions, the biggest vortices developed on the dayside (about $6 R_{\mathrm{E}}$ in diameter), had their rotation axes aligned with the $y$ direction and had the longest periods of duration. We attribute these vortices to the flows set up by reconnection on the highlatitude magnetopause during intervals of northward Interplanetary Magnetic Field (IMF) orientation. This is the first time that vortices due to high-latitude reconnection have been visualized. The model also successfully predicts the principal characteristics of previously reported plasma vortices within the magnetosphere, namely their dimension, flow velocities, and durations.
\end{abstract}

Keywords. Magnetospheric physics (MHD waves and instabilities)

\section{Introduction}

The solar wind-magnetosphere interaction can be steady or unsteady. Unsteady processes, like transient vortices at the magnetospheric boundary, may dominate the interaction by transferring mass, energy and momentum into the magnetosphere. Many processes have been suggested for the creation of such vortices, like pressure pulses, magnetic reconnection and the Kelvin-Helmholtz $(\mathrm{KH})$ instability (Sibeck et al., 2002; Shi et al., 2005; Miura, 1995; Kivelson and Chen, 1995). The latter is the most commonly cited for the creation of the vortices and occurs when surface waves are excited on the outer magnetospheric boundary by the velocity shear provided by magnetosheath plasma flowing past the magnetosphere.

Observations from single spacecraft, in conjunction with theoretical models and simulations, have frequently been used to explore and understand the KH instability (Ogilvie and Fitzenreiter, 1989; Chen et al., 1993; Otto and Fairfield, 2000; Hasegawa et al., 2006; Takagi et al., 2006; Fairfield et al., 2007; Collado-Vega et al., 2007). These studies explored the parameters favoring development of the instability. Some also addressed the transport of mass and momentum by the instability. Using multi-spacecraft observations, Hasegawa et al. (2004) employed Cluster observations to provide direct evidence for rolled-up vortices on the flank magnetopause during an interval of generally northward

Published by Copernicus Publications on behalf of the European Geosciences Union. 
Interplanetary Magnetic Field (IMF). Hasegawa et al. (2005) compared these observations with the output from threedimensional magnetohydrodynamic simulations for the solar wind's interaction with the magnetosphere. More recently, Palermo et al. (2010) presented KH vortex signatures detected by two of the five THEMIS spacecraft near the postnoon magnetopause.

Although the $\mathrm{KH}$ instability may be the best known instability capable of creating vortices near the magnetopause, other processes may also generate vortices. For example, plasma flows into the reconnection region, is accelerated, and flows outward. During intervals of southward IMF orientation, we expect reconnection on the dayside equatorial magnetopause. Magnetospheric plasma on closed magnetic field lines flows into the reconnection regions, while reconnected plasma on newly opened magnetic field lines flows antisunward along the magnetopause (Dungey, 1961). In the case of southward IMF near local noon, the flows would be in the meridional plane with an axis nearly in the $y$ direction.

By contrast, during intervals of strongly northward IMF, we expect IMF field lines to be appended to the magnetosphere via high latitude reconnection poleward of both cusps. There is flow into the reconnection site within the magnetosphere and the newly reconnected magnetic field lines move antisunward along the flanks of the magnetosphere (Song and Russell, 1992; Kessel et al., 1996; Song et al., 1999). This again results in flows with axes nearly in the $y$ direction. Both kinds of reconnection result in shears and large-scale plasma convection vortices (Saunders, 1989).

Indeed, vortices with solar wind properties very different to those predicted for the KH instability have definitely been observed. Zhang et al. (2011) reported vortical flows near the Low Latitude Boundary Layer (LLBL) in conjunction with flux transfer events (FTEs) during southward IMF. They discuss how antisunward moving FTEs transfer momentum to the LLBL plasma through a viscous interaction. This interaction drives antisunward flows in the LLBL along the sides of the FTEs, increasing the thermal pressure ahead of it, diverting the plasma normal to the magnetopause and then allowing it to return to locations where the pressure is low, forming a vortex. Flow vortices can also be related to field-aligned currents with possible corresponding auroral signatures (Sitar et al., 1998).

Collado-Vega et al. (2007) examined the output of MHD simulations driven by real solar wind input. They found that vortices generated under northward IMF were more prevalent on the dawnside than on the duskside, but were substantially less ordered on the dawnside than on the duskside. The scale sizes of most of the vortices were large, up to $10 R_{\mathrm{E}}$. Rotation axes were closely aligned with the $z$ direction in solar magnetic (SM) coordinate system. The vortices rotated preferentially clockwise on the dawnside, and counterclockwise on the duskside. These characteristics suggested an interpretation in terms of the KH instability.
A question arises as to whether such vortices would develop during intervals of steady solar wind conditions. To isolate cause and effect, this paper examines the magnetospheric response for fixed solar wind conditions, namely fixed density, temperature, and velocity. We compare and contrast the case for a nominal $360 \mathrm{~km} \mathrm{~s}^{-1}$ solar wind speed with one for a higher $700 \mathrm{~km} \mathrm{~s}^{-1}$ solar wind speed. In each case, we rotate the IMF direction from southward to northward as a step function $15 \mathrm{~min}$ into the run. We study how the IMF direction affects the growth and polarization of the vortices, and how fast the vortices develop depending on the velocity shear applied to the boundary. Based on our previous study, we predict that the vortices develop near the equatorial plane, with axes in the $z$ axis direction and having a sense of rotation that depends on location (clockwise on the dawnside and counterclockwise on the duskside). Some vortices did develop with these characteristics, but most of them had very different characteristics.

Section 2 describes the simulation and methods used for this study. Section 3 describes the vortices found near the magnetopause during fixed nominal solar wind conditions (case 1) and for high-speed solar wind conditions (case 2). Section 4 discusses and compares the vortices found in this study with those in the previously reported simulation for dynamic solar wind conditions (Collado-Vega et al., 2007). Closing remarks constitute Sect. 5 .

\section{Methodology}

\subsection{MHD simulation}

For this study we initiated the MHD simulation with a step function in the IMF direction; 15 min being southward, $-5 \mathrm{nT}$, and then turning northward, $+5 \mathrm{nT}$, for two hours. Two cases, one with a nominal speed solar wind $\left(360 \mathrm{~km} \mathrm{~s}^{-1}\right)$ and another with a high speed solar wind $\left(700 \mathrm{~km} \mathrm{~s}^{-1}\right)$, were run using the global MHD Block-Adaptive-Tree-SolarwindRoe-Upwind-Scheme (BATS-R-US) code (Powell et al., 1999; Gombosi et al., 2004; Tóth et al., 2012) developed at the Center for Space Environment Modeling at the University of Michigan. BATS-R-US is part of the Space Weather Modeling Framework, and was available to us by the Community Coordinated Modeling Center (CCMC).

BATS-R-US global magnetosphere (GM) solves 3-D MHD equations in finite volume form using numerical methods corresponding to Roe's approximate Riemann solver. It is coupled with the ionospheric electrodynamics solver that solves Poisson's equation. The code has a block-adaptive grid composed of three-dimensional rectangular blocks arranged in varying degrees of spatial refinement levels. It uses a grid with maximal resolution of $0.25 R_{\mathrm{E}}$ in the inner magnetosphere, the magnetosheath, the magnetopause and plasma sheet. The magnetospheric MHD part is attached to an ionospheric potential solver that provides electric 

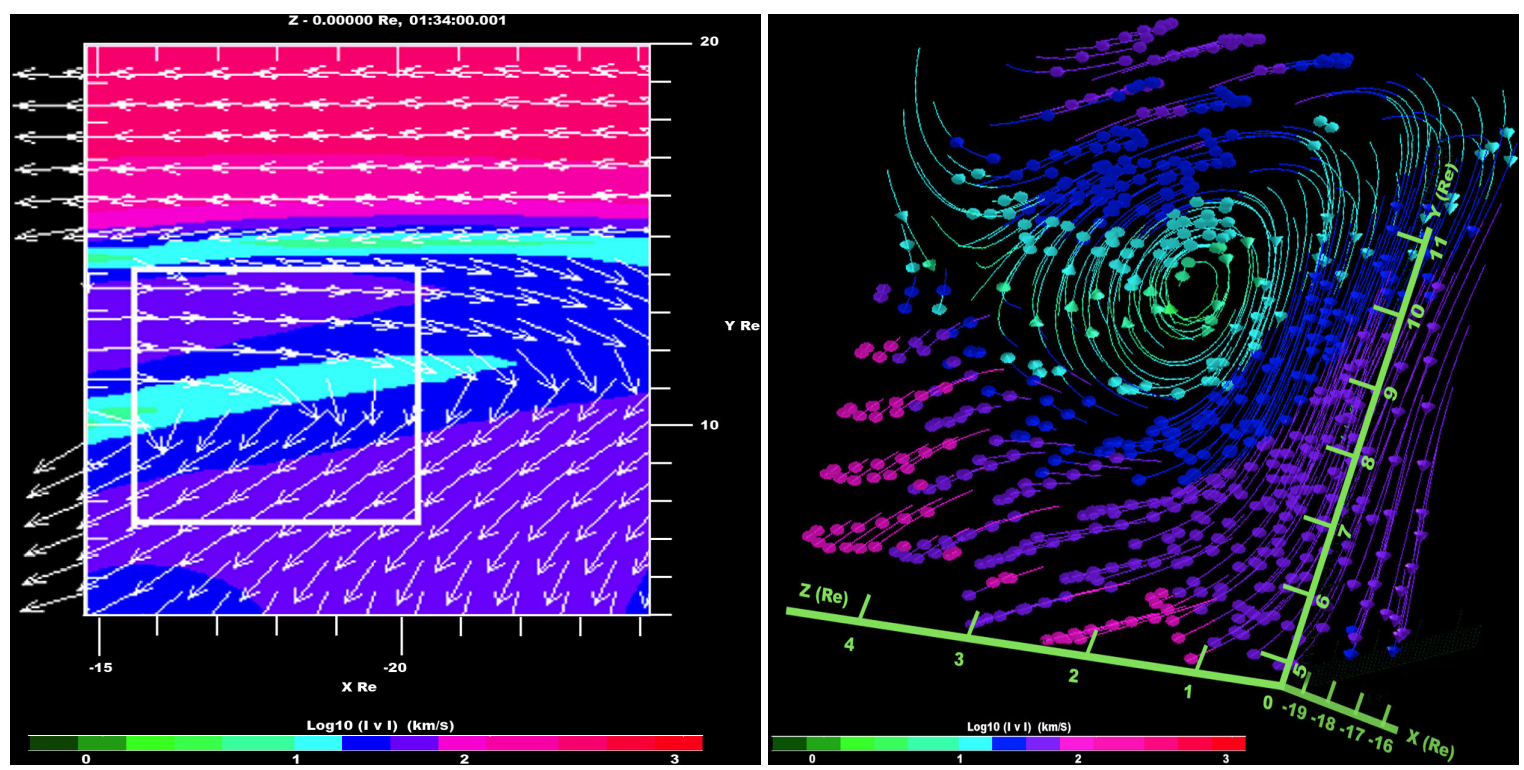

Fig. 1. Example of a vortex which would not be obvious with the naked eye, but that is correctly found with our algorithm. The colors represent the velocity magnitude in $\log$ scale and the arrows represent the flow vectors. The left panel is 2 -D in the $z=0$ cut plane on the duskside where the vortex is located. The right panel is the 3-D view of the vortex rotating approximately on the $x$ axis.

potentials and conductances in the ionosphere from magnetospheric field-aligned currents (Ridley et al., 2004). It uses local time stepping, which allows each grid cell to converge toward steady state as fast as possible, limited only by the local numerical stability limit.

The input parameters are transformed to geocentric solar magnetospheric (GSM) coordinates, and the Earth's magnetic field is approximated by a dipole with updated axis orientation and co-rotating inner magnetospheric plasma or with a fixed orientation during the entire simulation run. The orientation angle is updated according to the time simulated or a fixed axis position can be specified independently from the time interval that is simulated. In our simulation the dipole had a fixed orientation of 0 degrees for the entire simulation run, and a resolution of $0.4 R_{\mathrm{E}}$ was used on the dayside, which is small compared to the typical size of Kelvin-Helmholtz vortices $\left(\sim 3-10 R_{\mathrm{E}}\right)$.

In a previous study (Collado-Vega et al., 2007), statistics of vortices found near the ecliptic plane on the magnetopause flanks were presented using the Lyon-FedderMobbary (LFM) MHD simulation (Fedder et al., 1995a, b; Lyon et al., 2004). The simulation was performed in the solar magnetic (SM) coordinate system, allowing for the tilt of the Earth's magnetic dipole relative to the solar wind flow direction to be included. Outflow conditions are imposed on the downstream boundary. Elsewhere, external boundary conditions were specified using ACE solar wind data propagated appropriately to the front and cylindrical sides of the grid.

\subsection{Vortex identification and visualization}

Although the concept of a vortex is familiar, a rigorous definition has been elusive. Lugt (1995) suggested defining a vortex as "the rotating motion of a multitude of material particles around a common center". When viewing a vector field of velocity, we can use this definition to recognize vortices. However, quantitative measures are necessary to progress beyond labor-intensive manual identification. A measure of persistency is also intuitively part of the criteria, hence the link with coherent structures in a flow. A mathematical quantity, vorticity, is defined as the curl of the velocity vector $(\omega=\nabla \times \boldsymbol{v})$. It is a vector quantity whose direction is the axis of rotation, and is related to the local angular rate of rotation. Vorticity magnitude and isosurfaces are used to detect vortical structure successfully in some flows. The criterion of a local pressure minimum at a vortex arises from swirling flow arising from a balance between centrifugal force and pressure gradient, but this is not a sufficient condition and is problematic in 3-D.

An alternative way of viewing the flow is by its streamlines, the instantaneous curves that are everywhere tangent to the direction field defined by the velocity vectors. This leads to another qualitative definition for vortex identification: "A vortex exists when instantaneous streamlines mapped onto a plane normal to the vortex core exhibit a roughly circular or spiral pattern, when viewed from a frame of reference moving with the center of the vortex core" (Robinson, 1991). There are some difficulties associated with implementing this criterion, in particular it is not Galilean invariant, which means that whether or not the streamlines are 
Q1

Dawn Dayside

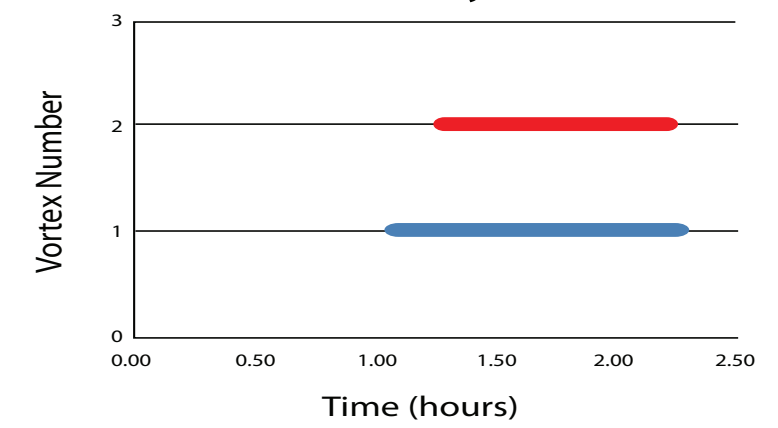

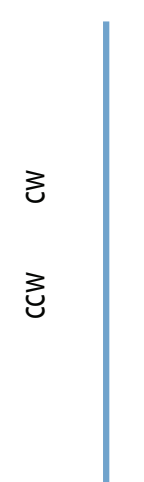
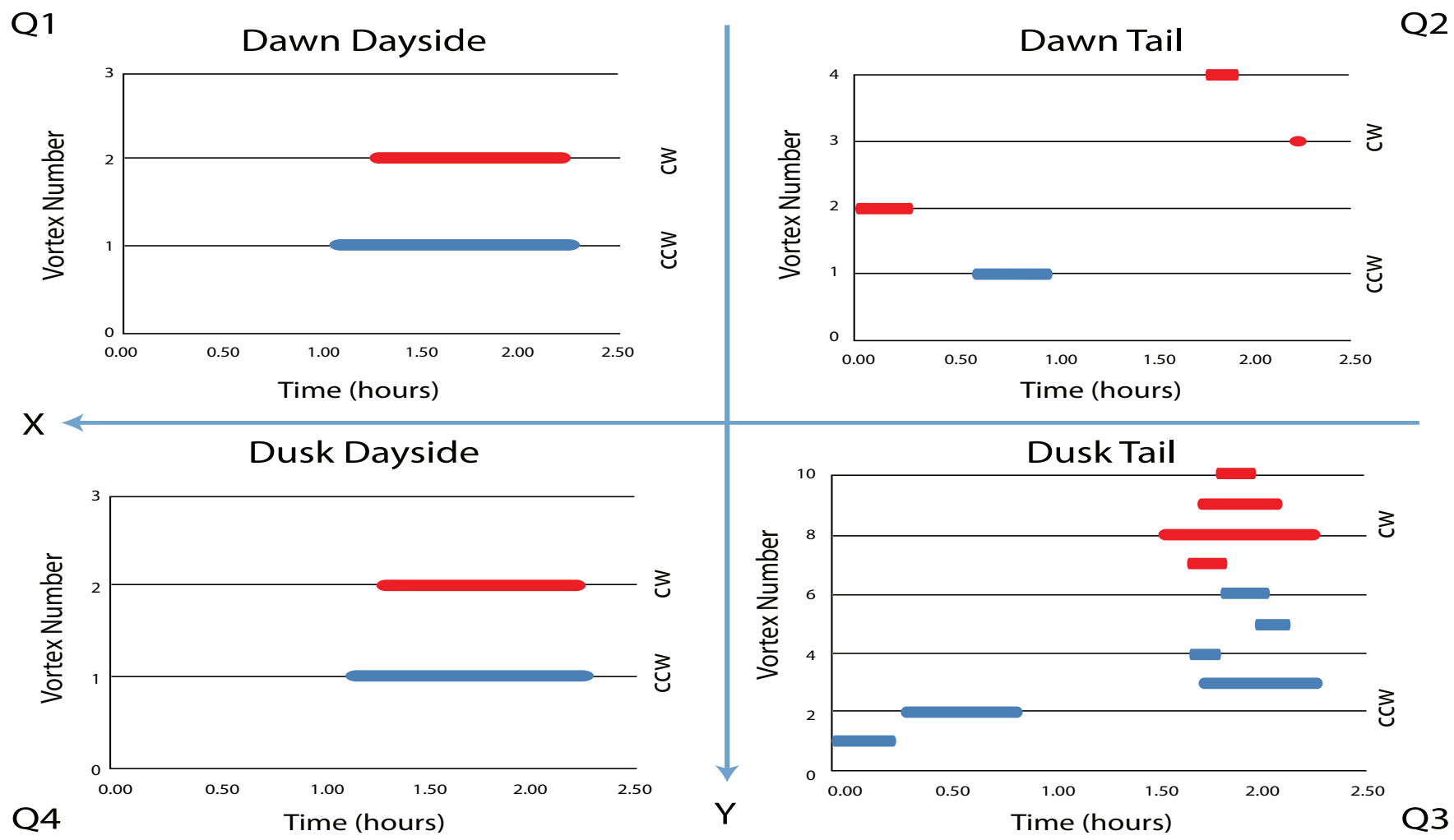

Fig. 2. Time duration of vortices for case 1. The $x$ axis represents the number of vortices and the $y$ axis represents the time in hours for each of the 4 quadrants. The blue $X$ and $Y$ arrows are just to show the separation in each of the four quadrants, $X$ positive being in the sunward direction and $Y$ positive in the duskward direction. The vortices rotating in the clockwise direction are represented in red color, and the ones rotating counterclockwise are represented in blue.

closed depends very much on one's frame of reference. Jeong and Hussain (1995) regard this as a requirement, although others, ourselves included, have had success in implementing this criterion.

Mathematical techniques adapted from topology have proven useful in elucidating structure in vector fields (Perry and Chong, 1994). Critical points are points in the flow field where the streamline slope is indeterminate and the velocity is zero relative to an appropriate observer. These points can be used to intuit important aspects of the vector field topology. These concepts may be more familiar in their use for magnetic field topology and visualization (Cai et al., 2001). In pursuit of vortices, the velocity gradient tensor is of particular interest. Let $\nabla v \boldsymbol{v}$ denote the velocity gradient tensor. Perry and Chong (1987) propose defining a vortex core as a region with complex eigenvalues of $\nabla \boldsymbol{v}$. The premise is that complex eigenvalues indicate closed streamlines in a reference frame moving with the point. $\nabla \boldsymbol{v}$ can be written as a sum of a symmetric part $S=\frac{1}{2}\left(\nabla \boldsymbol{v}+\nabla \boldsymbol{v}^{T}\right)$ and an antisymmetric part $\Omega=\frac{1}{2}\left(\nabla \boldsymbol{v}-\nabla \boldsymbol{v}^{T}\right)$. Then $Q=\frac{1}{2}\left(|\Omega|^{2}-|S|^{2}\right)$, the second invariant of the velocity gradient, is a measure of the shear strain rate and vorticity. Hunt et al. (1988) used the criterion of positive $Q$ to define eddies, along with low pressure. As Hunt et al. (1988) observed, for most vortices this criterion is equivalent to complex eigenvalues of the velocity gradient tensor. All these methods work well for some fluid flows, but our experience thus far with simulation data for the Earth's magnetosphere indicates that these measures are not sufficient to uniquely identify space plasma vortices.

Our current algorithm takes a multi-staged approach to locate vortices in 3-D flows. It requires only velocity components at each point in the domain. The second invariant of the velocity gradient tensor, $Q$, is used to identify points where the rotational strength exceeds the strain rate. The points that pass this test $(Q>0)$ are then subjected to further scrutiny. The vorticity vector is used to define the $\xi$-axis of a local coordinate system, and the velocity field is projected onto a plane perpendicular to this vector. Local streamlines are computed. A point is classified as a vortex if a small neighborhood exhibits both closed streamlines and a velocity magnitude minimum at the center. This algorithm is successful at finding the vortex center, but is not immune to false hits. More work is required to fine tune the search criteria and characterize both vortex size and strength. One method of screening for alleged eddies due to numerical effects is to cluster the vortices into classes by spatial proximity. The class size can be used as a filter to eliminate isolated points that are more likely to be numerical artifacts. It is far more 
Table 1. Solar wind plasma input parameters.

\begin{tabular}{ccc}
\hline Parameters & Case 1 & Case 2 \\
\hline$V_{x}$ & $-360 \mathrm{~km} \mathrm{~s}^{-1}$ & $-700 \mathrm{~km} \mathrm{~s}^{-1}$ \\
$V_{y}$ & $-28 \mathrm{~km} \mathrm{~s}^{-1}$ & $-28 \mathrm{~km} \mathrm{~s}^{-1}$ \\
$V_{z}$ & $-38 \mathrm{~km} \mathrm{~s}^{-1}$ & $-38 \mathrm{~km} \mathrm{~s}^{-1}$ \\
IMF $B_{x}$ & $2 \mathrm{nT}$ & $2 \mathrm{nT}$ \\
IMF $B_{y}$ & $1 \mathrm{nT}$ & $1 \mathrm{nT}$ \\
IMF $B_{z}$ & $-5 /+5 \mathrm{nT}$ & $-5 /+5 \mathrm{nT}$ \\
IMF |B| & $5.10 \mathrm{nT}$ & $5.48 \mathrm{nT}$ \\
IMF clock angle & $168.7 \mathrm{deg}$ & $168.7 \mathrm{deg}$ \\
Density & $7 \mathrm{ncc}^{-1}$ & $3 \mathrm{ncc}^{-1}$ \\
Temperature & $40000 \mathrm{~K}$ & $40000 \mathrm{~K}$ \\
\hline
\end{tabular}

likely that a genuine vortex is part of a vortex tube that has a spatial extent. Figure 1 shows an example of a vortex which would not be obvious with the naked eye, but is correctly found with our algorithm. At first, the visualization tool only shows 2-D cut planes of a box with dimensions of $x=-25$, $25 R_{\mathrm{E}}, y=-20,20 R_{\mathrm{E}}$. The vortex algorithm provides the location from which to begin so the locations of interest are identified faster, and can be visualized in the 3-D view. The left-hand image shows a cut plane from a data simulation (cropped, near the tail at the magnetosphere) with $z=0$. The vectors show the $x$ and $y$ components of the velocity vector and the colors represent the velocity magnitude in a logarithm scale. The right-hand image shows the 3-D velocity field for the data within the white square in the left-hand image.

Although our vortex algorithm is very successful at identifying individual vortices, it is less successful at grouping vortices both temporally and spatially. In order to determine whether individual vortices were really part of the same vortex, for example, persisting at one location for an extended time or convecting tailward over time, two additional methods were employed. The MHD Explorer tool discussed in Collado-Vega et al. (2007), was used to visualize both individual vortices and groups of vortices. The human eye is capable of discerning collections and patterns for which computer algorithms do not yet exist. Secondly, Excel spreadsheets were used to group similar characteristics (axes of rotation, rotation direction, diameter, etc.), determine persistence in time, and to plot vortex locations after each vortex was carefully studied by the naked eye. The resulting collected vortices are discussed in the following sections.

\section{Vortices under fixed solar wind conditions}

In Table 1 we describe two sets of input parameters for the MHD simulation. For each case we fixed the solar wind velocity $\left(V_{x}, V_{y}, V_{z}\right)$, IMF $\left(B_{x}, B_{y}, B_{z}\right)$, IMF clock angle, density, and temperature. Each simulation ran for a total of $2 \mathrm{~h}$ and $15 \mathrm{~min}$. For both cases the IMF $B_{z}$ component was

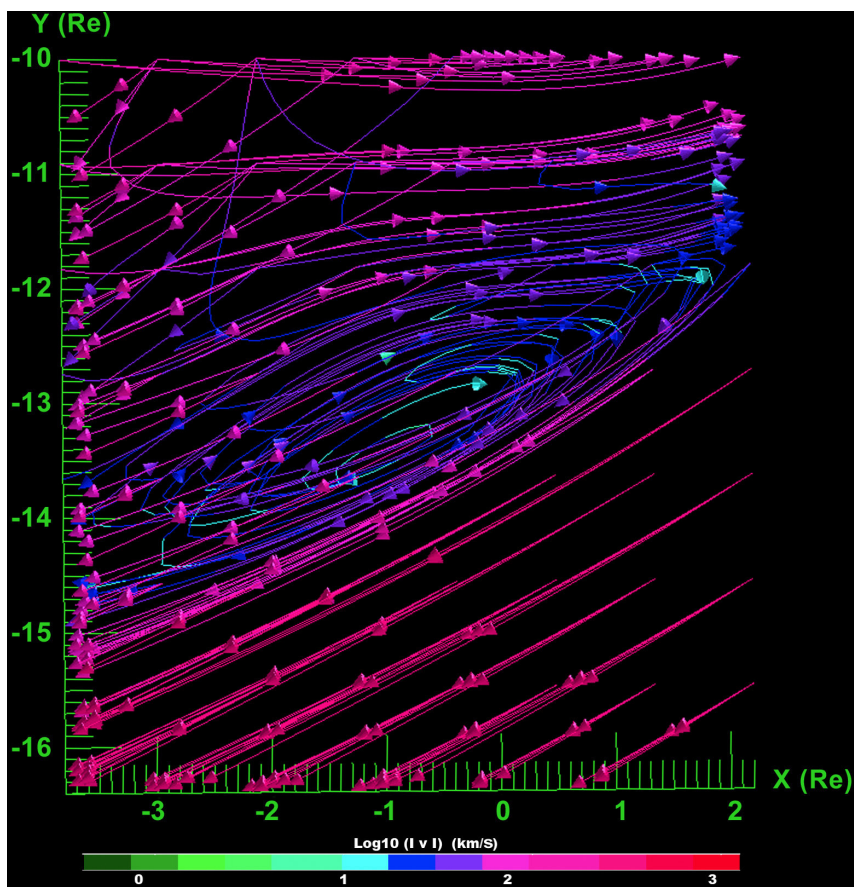

Fig. 3. Dawnside vortex rotating clockwise with its rotation axis aligned in the $z$ direction (pattern 1) that developed during southward IMF within the first 15 min of the simulation run.

initially southward for the first $15 \mathrm{~min}$, and then turned northward with the same magnitude ( $5 \mathrm{nT}$ ) for the rest of the simulation. In case 1 the $x$ component of the solar wind speed was nominal with a value of $-360 \mathrm{~km} \mathrm{~s}^{-1}$. The transverse components had significantly lower values $\left(V_{y}=-28 \mathrm{~km} \mathrm{~s}^{-1}\right.$ and $V_{z}=-38 \mathrm{~km} \mathrm{~s}^{-1}$ ), the density was $7 \mathrm{ncc}^{-1}$ and the temperature had a value of $40000 \mathrm{~K}$. The IMF transverse components had small values: $B_{x}=2 \mathrm{nT}$ and $B_{y}=1 \mathrm{nT}$, and the IMF clock angle was 168.7 degrees. For the high solar wind speed case $\left(V_{x}=700 \mathrm{~km} \mathrm{~s}^{-1}\right)$, the density had a lower value of $3 \mathrm{ncc}^{-1}$, in keeping with observations of typical solar wind trends. The $x$ and $y$ components of the IMF and the clock angle were the same as in case 1 .

Using the automated vortex detection algorithm, spreadsheets, and the MHD Explorer visualization tool with the output parameters of the BATS-R-US simulation runs, a total of 33 vortices were found for the two cases with fixed solar wind conditions; 18 vortices were found for the slower solar wind speed case, and 15 vortices were found for the faster solar wind speed case. The specifics are discussed in the next two sections.

\subsection{Nominal solar wind speed: case 1}

During the $2 \mathrm{~h}$ and $15 \mathrm{~min}$ simulation run, vortices developed throughout the simulation in two distinct patterns: (1) vortices with their rotation axis in the $z$ direction, and (2) vortices with their rotation axis in either the $x$ or $y$ direction. The 


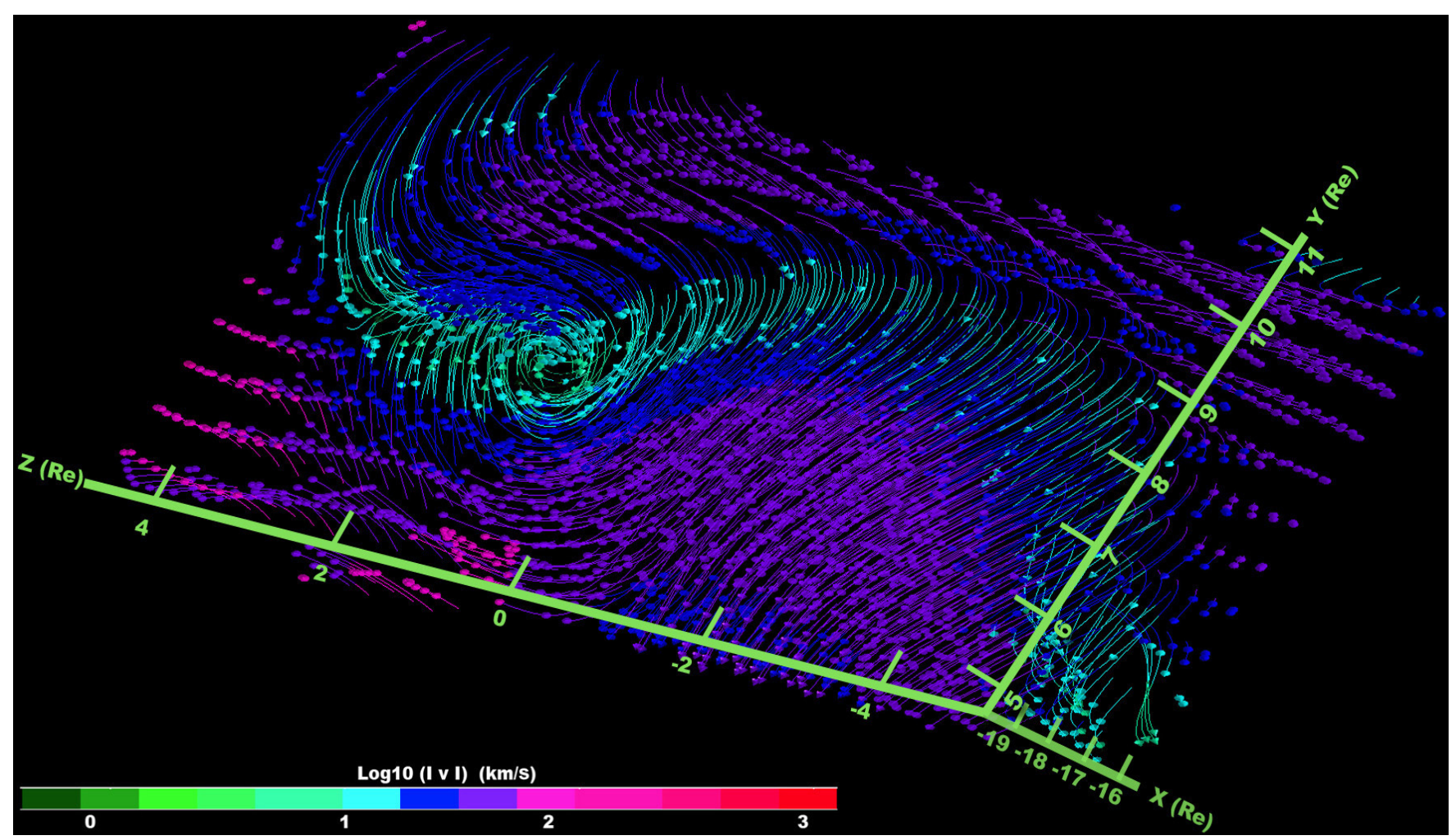

Fig. 4. Dusk nightside vortex that shows how some vortices could be rotating on the $x$ axis and then twisting into another direction.

first pattern includes vortices that developed during the first 15 min with southward IMF, vortices that developed early on when the IMF turned northward, and vortices that developed much later in the simulation. For the first pattern, vortices developed along both the dawn and dusk flank; those on the dawnside rotated in the clockwise direction, while those on the duskside rotated preferentially in the counterclockwise direction (of four cases, three rotated in the counterclockwise direction). For the second pattern, vortices were not as wellordered and could twist so that part of a vortex would have a rotation axis in the $x$ direction, and another part of the same structure would have its rotation axis in the $y$ or $z$ direction. Their rotation directions were both clockwise and counterclockwise, not dependent on location. These formed more than thirty minutes after the simulation began when conditions were steady. Figure 2 shows vortices found in each of the four quadrants, along with their duration in the simulations. The dusk magnetotail had many more vortices than the other quadrants. Vortices on the dayside typically lasted longer than on the nightside. All of the vortices are described in more detail below.

During the first $15 \mathrm{~min}$ of the simulation when the IMF was southward, only 2 vortices developed, as shown in Fig. 2, quadrants 2 and $3(\mathrm{Q} 2, \mathrm{Q} 3)$. One vortex developed on the dawn tail flank, $x=-0.79 R_{\mathrm{E}}, y=-13 R_{\mathrm{E}}$, and the other developed on the dusk tail flank, $x=-2.76 R_{\mathrm{E}}, y=$ $12.72 R_{\mathrm{E}}$. Both of the vortices fit the first pattern, with their rotation axis closely aligned to the $z$ direction. In Fig. 3 we show the vortex that developed on the dawnside. Its rotation axis is closely aligned parallel to the positive $z$ axis and it rotates in the clockwise direction, where the direction of rotation for all the vortices is always chosen when viewed from the positive coordinate axes. The vortex that developed at the duskside (not shown) rotated in the counterclockwise direction. No vortices developed on the dayside during the $15 \mathrm{~min}$ of southward IMF (Q1 and Q4 in Fig. 2).

Ten minutes after the IMF turned northward, one vortex fully developed (Q3 in Fig. 2). This was along the dusk tail flank, with a rotation axis in the $z$ direction and counterclockwise rotation. This vortex lasted $30 \mathrm{~min}$ and slowly convected down tail. On the dawn side tail flank (Q2), a pattern 1 vortex developed and then dissipated, with another one developing at about the same position $10 \mathrm{~min}$ later and then convecting down tail. This vortex also had its axis in the $z$ direction, but rotated clockwise. After about one and a half hours of simulation time, a few more vortices that fit the first pattern developed in the dusk tail. These were short lived and found in the vicinity of vortices of the second pattern.

Pattern 2 vortices were found in all four quadrants. On the dayside they formed after about an hour of simulation time and persisted for the rest of the simulation. The dayside pattern 2 vortices had their rotation axes in the $y$ direction; there was one clockwise and one counterclockwise in both dayside 


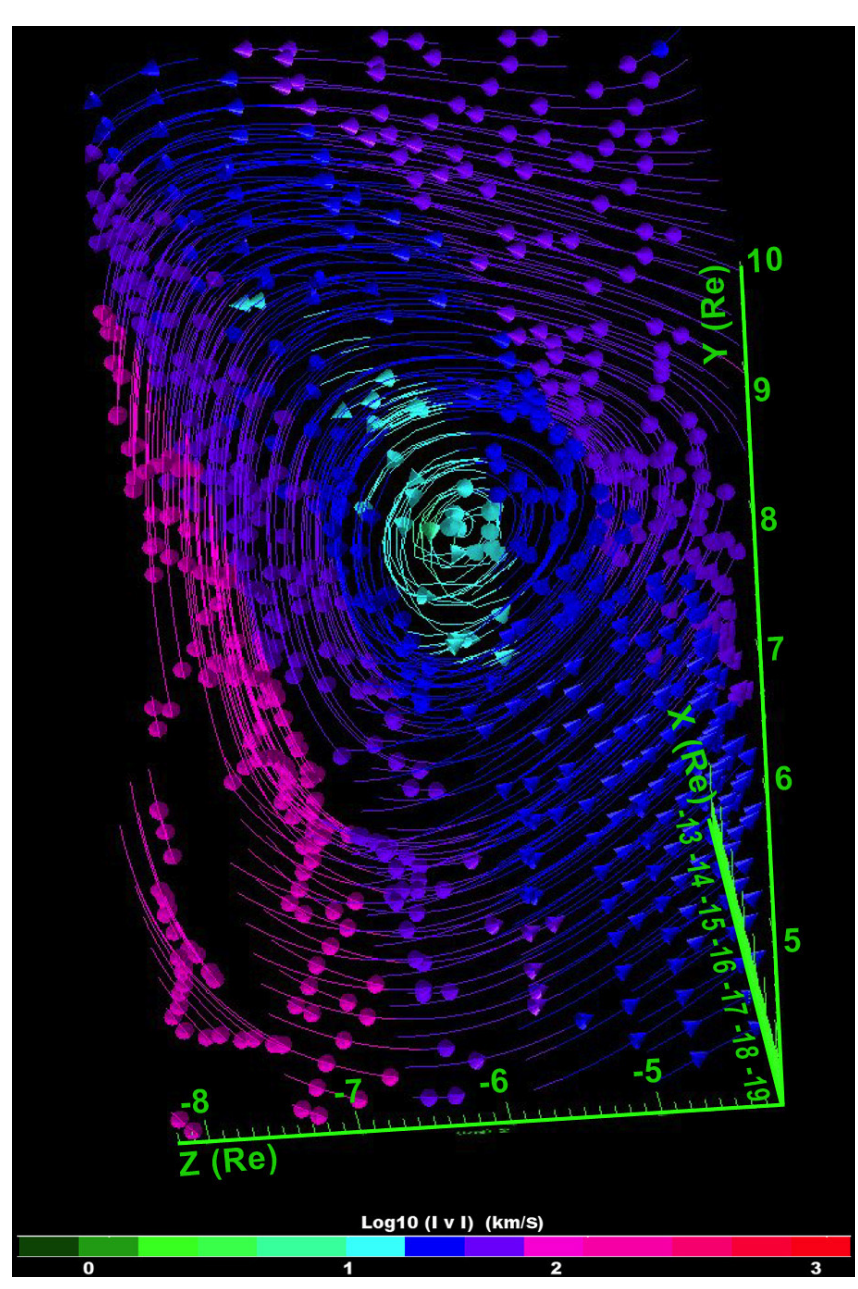

Fig. 5. Inner dusk vortex rotating counterclockwise with its axis closely aligned with the $x$ direction (pattern 2).

quadrants (Q1 and $\mathrm{Q} 4$ in Fig. 2). These vortices formed in the $z=-5.6 R_{\mathrm{E}}$ cut plane.

On the nightside, the pattern 2 vortices were more likely to be oriented in the $x$ direction but could twist into another direction. An example is shown in Fig. 4. Here a dusk nightside vortex formed around one hour and 30 min of the simulation run with one part rotating on the $x$ axis, and then turning into the $z$ axis. On the dawnside the two pattern 2 vortices rotated in the clockwise direction. On the dusk side pattern 2 vortices were found with both rotation directions, clockwise and counterclockwise. In Fig. 5 a nightside dusk vortex is rotating counterclockwise with its axis closely aligned with the $x$ direction.

Table 2 lists the characteristics of the vortices that developed for the two patterns in each quadrant. This table shows that the dayside vortices were significantly larger than the nightside vortices, $6 R_{\mathrm{E}}$ on the dayside compared to $2-4 R_{\mathrm{E}}$ on the nightside. The table summarizes the characteristics that were described above.

\subsection{High solar wind speed: case 2}

For the high solar wind speed case, case 2, a total of 15 vortices formed. As for case 1, vortices also developed in the same two distinct patterns. The first pattern formed with the vortex axes in the $z$ axis direction, and the second pattern formed by the vortex axes in the $x-y$ direction. Figure 6 shows the vortices that formed under case 2 in all four quadrants with their time duration.

During the first $15 \mathrm{~min}$ of the simulation, when the IMF was completely southward, only two vortices formed. They both developed at the beginning of the simulation (Fig. 6 Q2 and Q3). One developed at the dawn tail flank (Q2) and the other at the dusk tail flank (Q3). The dawnside vortex rotated clockwise, and the duskside vortex rotated counterclockwise. Both vortices had their rotation axis aligned to the $z$ axis. As in case 1, no vortices developed on the dayside region for the southward IMF. After the 15 min of southward IMF had passed, only one more vortex developed with its axis closely aligned to the $z$ axis (pattern 1). It developed at around thirty minutes before the end of the simulation at the dawn tail flank and moved towards the boundary $(-z)$ very slowly until the end of the simulation.

Pattern 2 vortices started developing after $35 \mathrm{~min}$ of the IMF being northward. These vortices developed at the dawn and dusk dayside with their rotation axis closely aligned to the $y$ axis (Fig. 6, Q1 and Q4). They were the bigger sized vortices developed for this case, with about $6 R_{\mathrm{E}}$ of diameter. They are also seen until the end of the simulation run, lasting for about $1 \mathrm{~h}$ and $25 \mathrm{~min}$. Some vortices also developed at the dawn and dusk nightside region. However, more vortices formed at the dawnside for case 2 . These vortices had the rotation axis closely aligned to the $x$ axis. They were mostly about $3-4 R_{\mathrm{E}}$ in diameter, and lasted around $30-45 \mathrm{~min}$. No preference in rotation was noted for the vortices of pattern 2 . These vortices' durations can be seen in quadrants 2 and 3 in Fig. 6.

Also, two vortices in pattern 2 developed simultaneously at the same $x-y$ location but with a different $z$ coordinate, and they rotate in the opposite sense. In Fig. 7 the vortices are shown having approximately the same $x-y$ location, but with different $z$ coordinates on the dusk dayside. One of the vortices rotates clockwise, and the other rotates counterclockwise. Vortices also appeared to have symmetry in which dawn and dusk vortices would develop simultaneously on the magnetopause flanks with different rotation directions. Figure 8 presents a 2-D cut plane with $z=-5.6 R_{\mathrm{E}}$ where a dawn and a dusk vortex developed simultaneously rotating counterclockwise (dusk) and clockwise (dawn).

Table 3 lists the characteristics of the vortices formed for case 2 . It shows that the dayside vortices were bigger $\left(6 R_{\mathrm{E}}\right)$ than the biggest ones formed at the nightside $\left(3-4 R_{\mathrm{E}}\right)$. Also, it shows that for this case the majority of the vortices were formed at the dawnside, not at the duskside like in case 1. 


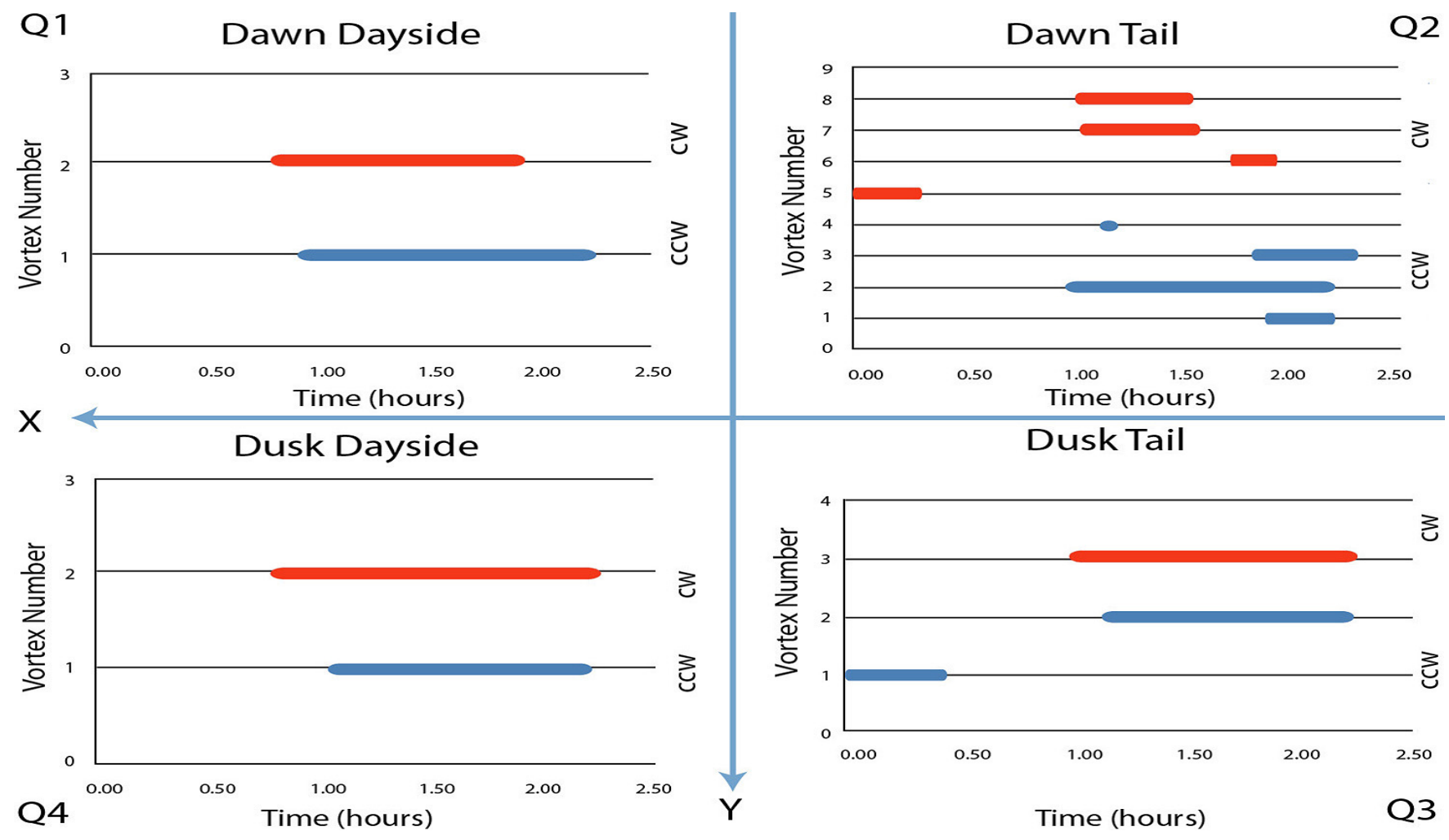

Fig. 6. Same as Fig. 2, but for the vortices found for case 2 (higher solar wind speed). The vortices rotating clockwise are represented in red color and those rotating counterclockwise are represented in blue.

Table 2. Case 1: four quadrant vortex statistics.

\begin{tabular}{|c|c|c|c|c|c|c|c|c|}
\hline \multirow[t]{2}{*}{ Pattern } & \multicolumn{4}{|c|}{ Dawn dayside } & \multicolumn{4}{|c|}{ Dawn nightside tail } \\
\hline & \# & Axis & Rotation & Size & $\#$ & Axis & Rotation & Size \\
\hline 1 & 0 & & & & 2 & $z$ & $\mathrm{cW}$ & $3-4 R_{\mathrm{E}}$ \\
\hline 2 & 2 & $y$ & $\mathrm{ccw}, \mathrm{cw}$ & $6 R_{\mathrm{E}}$ & 2 & $x$ & $\mathrm{cw}$ & $3-4 R_{\mathrm{E}}$ \\
\hline \multirow[t]{2}{*}{ Pattern } & \multicolumn{4}{|c|}{ Dusk dayside } & \multicolumn{4}{|c|}{ Dusk nightside tail } \\
\hline & \# & Axis & Rotation & Size & $\#$ & Axis & Rotation & Size \\
\hline 1 & 0 & & & & 4 & $z$ & $\mathrm{ccw}, \mathrm{cw}$ & $2-4 R_{\mathrm{E}}$ \\
\hline 2 & 2 & $y$ & $\mathrm{ccw}, \mathrm{cw}$ & $6 R_{\mathrm{E}}$ & 6 & $x$ & $\mathrm{ccw}, \mathrm{cw}$ & $2-4 R_{\mathrm{E}}$ \\
\hline
\end{tabular}

\section{Discussion and comparison}

This study explored vortices developed in an MHD simulation with fixed solar wind conditions, while a previous study (Collado-Vega et al., 2007) looked at dynamic solar wind conditions shown in Fig. 9. Using the new search algorithm described in Sect. 2 we re-examined data from this previous study, but only times for which the conditions were similar to the current study, with a dynamic pressure of about 5-10 nPa. The magnetic field, however, was greater, $10-15 \mathrm{nT}$ for one interval and 20-25 $\mathrm{nT}$ for the second interval (cases $\mathrm{C}$ and $\mathrm{E}$ from Collado-Vega et al., 2007). The most significant differ- ences between the vortices formed with dynamic solar wind conditions and the ones formed under fixed solar wind conditions are rotation (preference and axis), convection, location, time of duration and other characteristics as described below.

\subsection{Rotation}

In the current study, pattern 1 vortices for both case 1 and case 2 had axes aligned with the $z$ direction and rotated preferentially clockwise on the dawnside, and counterclockwise on the duskside. For both cases, a total of 4 pattern 1 vortices were found on the dawn nightside, from which 3 rotated in the clockwise direction, and a total of 5 pattern 1 vortices 


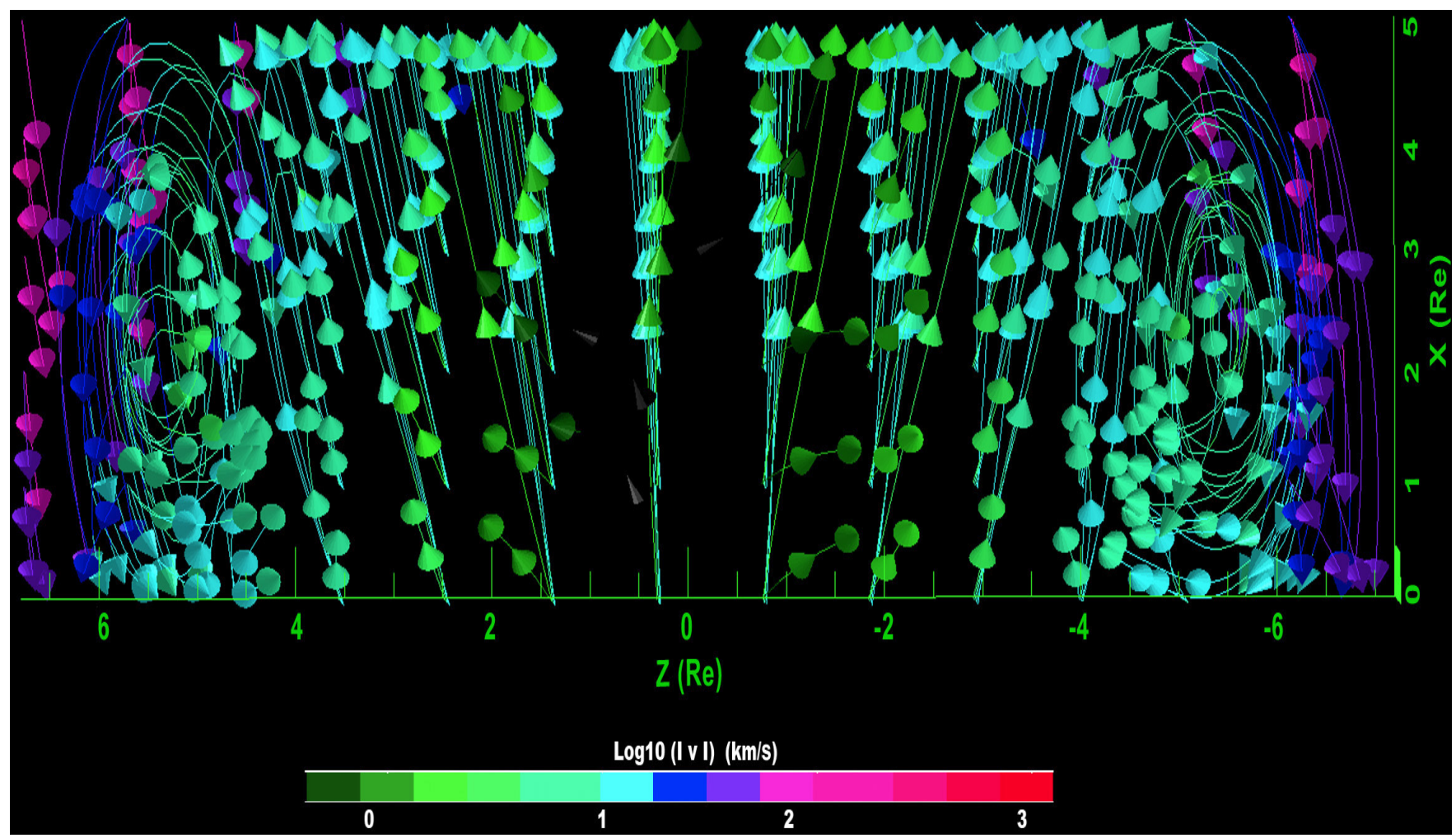

Fig. 7. Vortices on the dusk dayside. The vortices formed almost at the same $x-y$ location, but with a different $z$ coordinate. This is in the $y=7 R_{\mathrm{E}}$ cut plane.

Table 3. Case 2: four quadrant vortex statistics.

\begin{tabular}{|c|c|c|c|c|c|c|c|c|}
\hline \multirow[t]{2}{*}{ Pattern } & \multicolumn{4}{|c|}{ Dawn dayside } & \multicolumn{4}{|c|}{ Dawn nightside tail } \\
\hline & $\#$ & Axis & Rotation & Size & \# & Axis & Rotation & Size \\
\hline 1 & 0 & & & & 2 & $z$ & $\mathrm{ccw}, \mathrm{cw}$ & $3 R_{\mathrm{E}}$ \\
\hline 2 & 2 & $y$ & $\mathrm{ccw}, \mathrm{cw}$ & $6 R_{\mathrm{E}}$ & 6 & $x$ & $\mathrm{ccw}, \mathrm{cw}$ & $3-4 R_{\mathrm{E}}$ \\
\hline \multirow[t]{2}{*}{ Pattern } & \multicolumn{4}{|c|}{ Dusk dayside } & \multicolumn{4}{|c|}{ Dusk nightside tail } \\
\hline & $\#$ & Axis & Rotation & Size & \# & Axis & Rotation & Size \\
\hline 1 & 0 & & & & 1 & $z$ & $\mathrm{ccw}$ & $3 R_{\mathrm{E}}$ \\
\hline 2 & 2 & $y$ & $\mathrm{cw}, \mathrm{ccw}$ & $6 R_{\mathrm{E}}$ & 2 & $x$ & $\mathrm{cw}$ & $1-4 R_{\mathrm{E}}$ \\
\hline
\end{tabular}

were found on the dusk nightside, from which 4 rotated in the counterclockwise direction. Pattern 2 vortices had rotation axes mostly aligned with the $x$ or $y$ axis. On the dayside, case 1 and case 2 pattern 2 vortices had rotation axes aligned with the $y$ axis, but on the nightside their rotation axes were aligned with the $x$ axis. No preference in rotation direction was evident for pattern 2 vortices. In the previous dynamic study, vortices developed with rotation axes in the $z$ axis. In this study these would be classified as pattern 1 vortices. However, in the re-examination of the two similar cases with our new automated algorithm, 71 vortices were found with rotation axes aligned with the $x$ or $y$ direction.
These would be classified as pattern 2 vortices in our current study. Figure 10 shows a dawn vortex of 30 March 2002 at 00:40 UT corresponding to the higher magnetic field case of the dynamic conditions for the previous study. This vortex has its rotation axis closely aligned to the $x$ axis.

In the previous study, pattern 1 vortices rotated preferentially clockwise on the dawnside and counterclockwise on the duskside as was seen for the pattern 1 vortices in our current study. This behavior was attributed to the polarization of surface waves that could be driven by $\mathrm{KH}$ instability at the magnetopause (Collado-Vega et al., 2007). In our current study, more pattern 2 vortices developed than pattern 1 for 


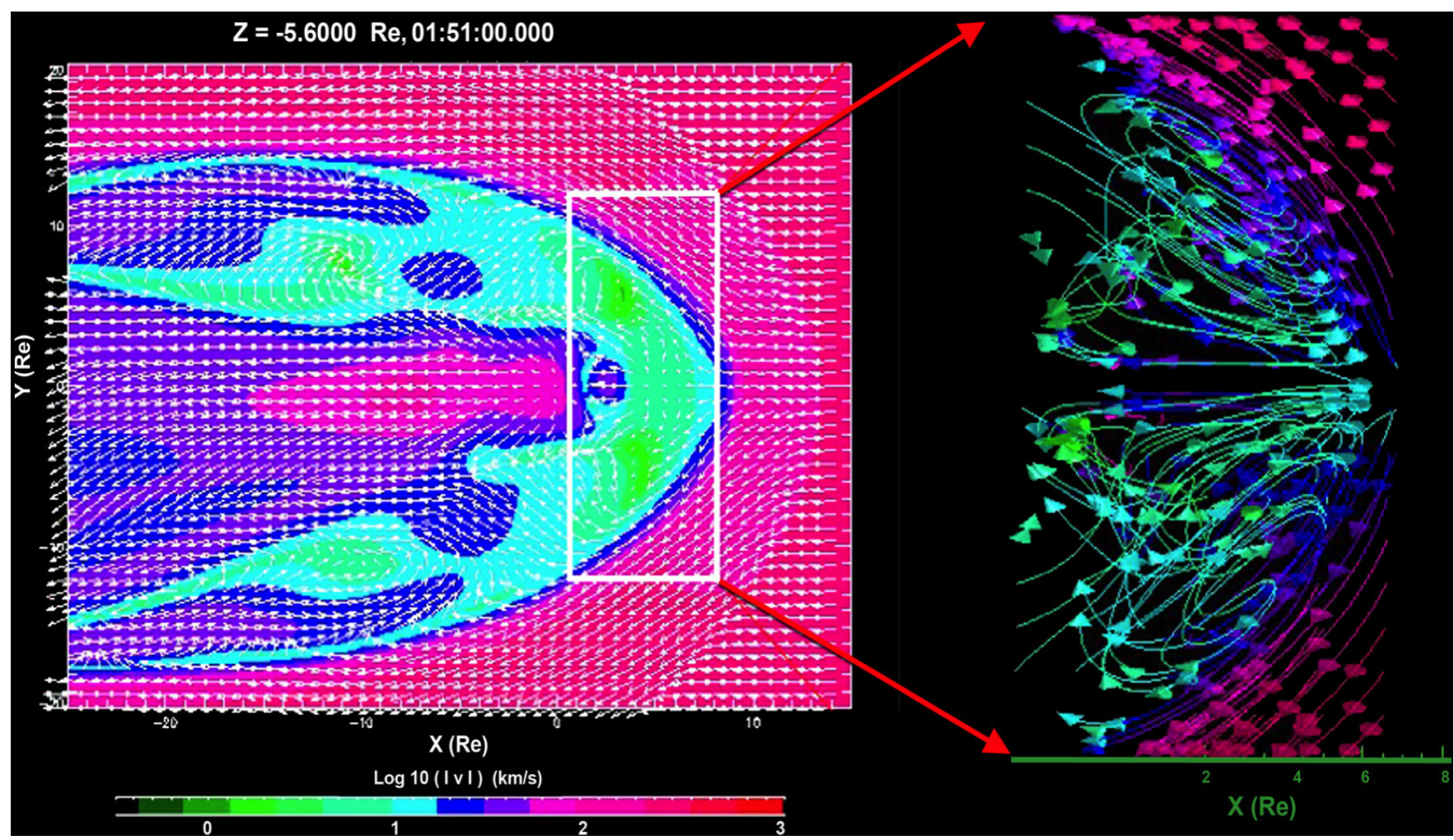

Fig. 8. Dawn and dusk vortices found simultaneously rotating counterclockwise(dawn) and clockwise(dusk). These vortices came in pairs with the ones shown in Fig. 7. The rotation direction is determined by following the arrowheads from the streamlines from the positive $y$ axis. The vortices are located in the same $z=-5.6 R_{\mathrm{E}}$, but the dawn dayside on $x=3.8 R_{\mathrm{E}}, y=-7.2 R_{\mathrm{E}}$, and the dusk dayside on $x=2.6 R_{\mathrm{E}}$, $y=6.8 R_{\mathrm{E}}$

both case 1 and case 2 . The axes of these vortices tended to lie parallel (or antiparallel) to the magnetospheric magnetic field, and they also developed during times of stable solar wind conditions contrary to our previous study where vortices formed under dynamic solar wind conditions.

Figure 11 shows the rotation polarity for all the vortices that exhibited rotation axes either parallel (counterclockwise when viewed from the dominant magnetic field axis) or antiparallel direction (clockwise when viewed from the dominant magnetic field axis) to the dominant magnetic field component. The sense of rotation therefore follows the righthand rule. Dayside vortices follow a pattern with the ones on the duskside rotating parallel to the local magnetic field, and those on the dawnside rotating antiparallel. We understand this rotation in terms of the global convection pattern and reconnection: plasma on closed magnetospheric magnetic field lines moves sunward through the dayside magnetosphere, while plasma on open magnetospheric magnetic field lines moves antisunward. No definite pattern was seen for the vortices that form on the nightside. However, more vortices rotate parallel to the magnetic field on the dawnside than on the duskside. More statistics need to be considered to reach a final decision about the nightside vortices. The black curve shows the magnetopause location as calculated by the BATS-
R-US model for the last time step in case 2. This shows that all the vortices were found inside the magnetosphere.

\subsection{Convection}

In the current study there were several pattern 1 vortices $(z$ direction axis) that convected tailward. These vortices occurred mostly during times when the solar wind conditions had just changed: at the beginning of the simulation and soon after the IMF switched from southward to northward. In case 1 there were four convecting vortices: two each for southward and northward IMF, on each tail flank. For case 2 only two convecting pattern 1 vortices formed, one on each dawn and dusk flank during southward IMF. In the previous study pattern 1 vortices were consistently swept antisunward by convection, becoming larger in diameter and changing velocity as they traveled along the flanks (Collado-Vega et al., 2007). As an example, in Fig. 12, a clear progression in vortex development is shown. The vortex is traveling antisunward along the dusk flank. Within only four minutes (02:4602:49 UT), the vortex core travels approximately $12 R_{\mathrm{E}}$ down the flank. In the re-examination of the two cases from the previous study, the pattern 2 vortices that were found appeared to be convecting down tail; however, their time duration was 


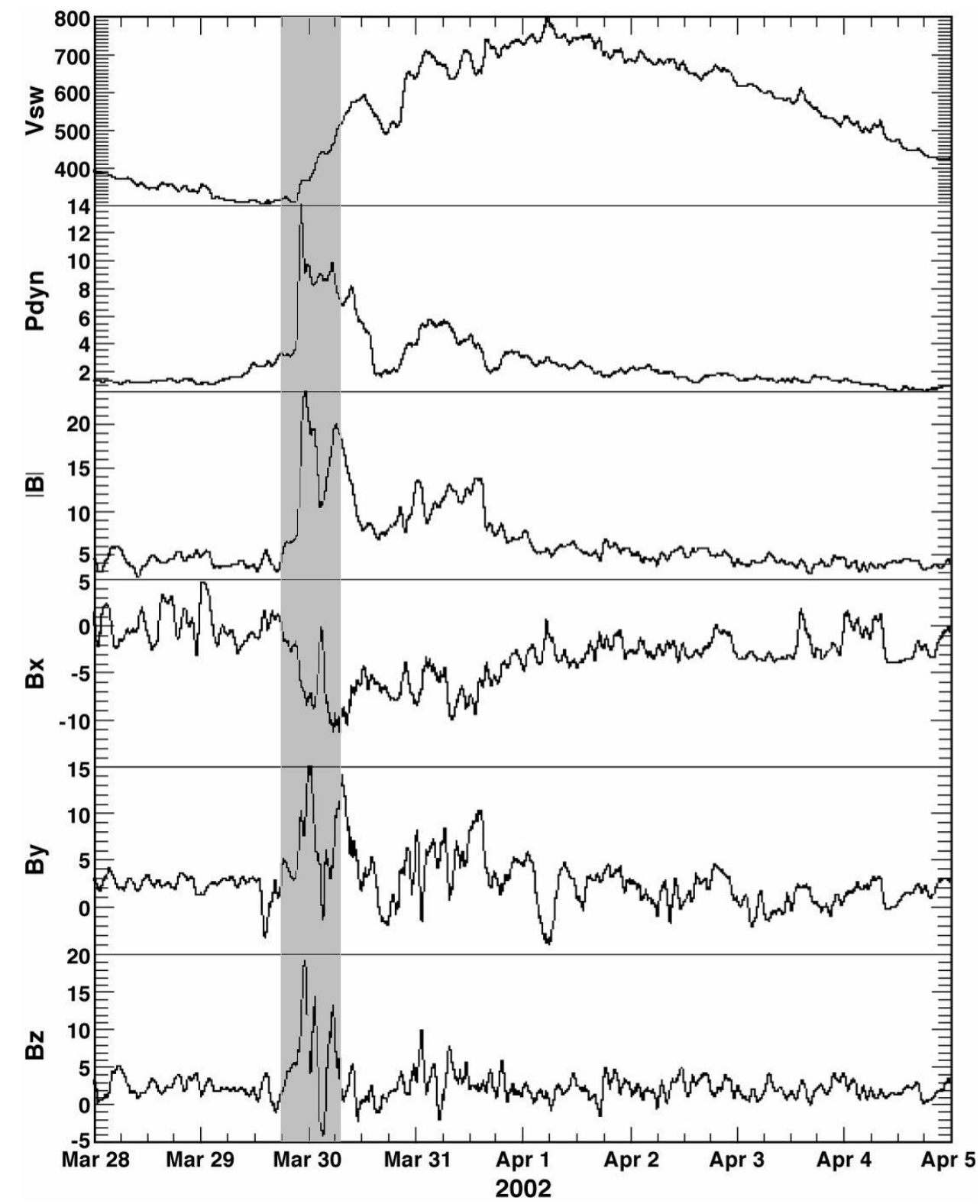

Fig. 9. Average values for the high-speed solar wind stream from 28 March to 5 April 2002. The shaded region shows the nine hour time frame of the vortices searched in Collado-Vega et al. (2007).

in terms of seconds. The longest duration vortices found only lasted for about $2 \mathrm{~min}$. These characteristics will be described in more detail below.

Most of pattern 1 vortices convected tailward for our current study. We found a few others that did move towards the boundary in the negative $z$ direction. Once steady state conditions were established, convection disappeared. We found three more vortices for case 2 pattern 2 that moved but in the negative $z$ direction also. The vortices that convected tailward did it more slowly than vortices in the previous study, taking up to $30 \mathrm{~min}$ to convect down tail out of the simulation box. The rest of the vortices formed for case 1 and case 2 did not convect. They had fixed positions, and lasted longer periods of time than those that did convect. These results suggest that it takes a change in solar wind conditions to generate vortices that convect tailward, but the change can be a sharp step function. The pattern 2 vortices found in the reexamination appeared to convect tailward, but because most of them lasted just seconds, and since we are looking at one minute plasma date files with our visualization tool, it is difficult to be completely sure about in which direction they are moving. 


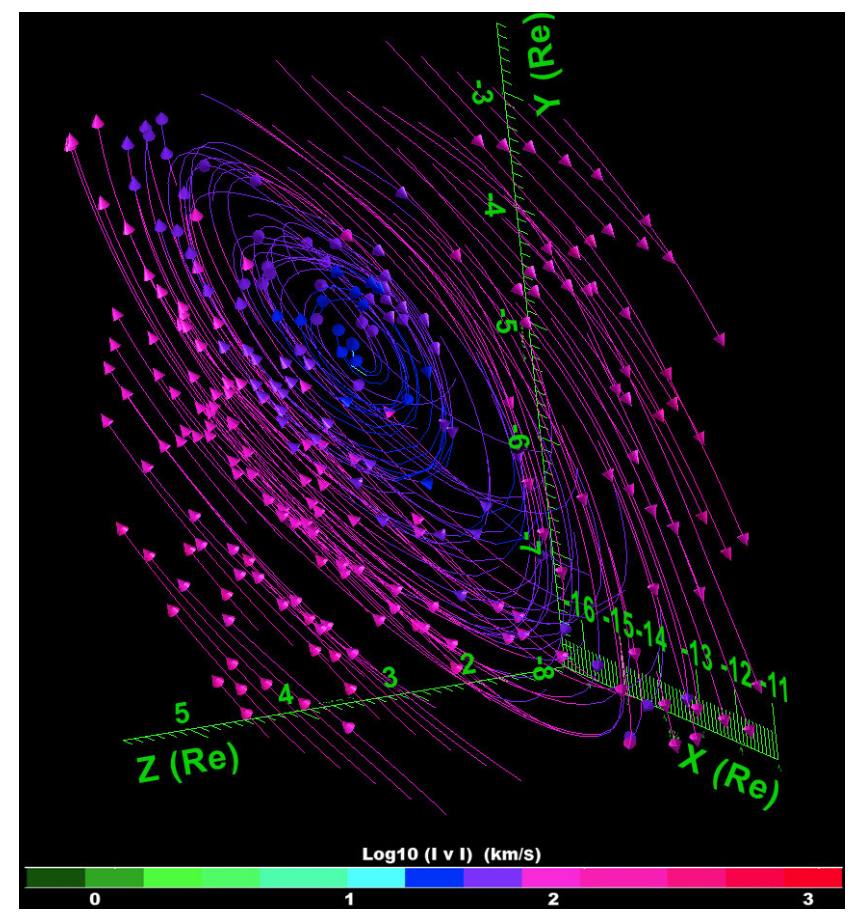

Fig. 10. Dawn vortex of the previous study case from Collado-Vega et al. (2007) of 30 March 2002, 00:40 UT, that was found using our automated search algorithm with its rotation axis closely aligned with the $x$ axis.

\subsection{Location}

In our current study, pattern 1 vortices tended to be near the equatorial dawn and dusk flanks on the nightside, for both convecting and non-convective vortices. This is also true for the vortices found in our previous study. No pattern 1 vortices developed on the dayside. Pattern 2 vortices developed in all 4 quadrants; however, more developed on the dusk nightside for case 1 and more on the dawn nightside for case 2. Pattern 1 vortices generated under northward IMF for the previous study (Collado-Vega et al., 2007) were more prevalent on the dawnside than on the duskside, and were substantially less ordered on the dawnside than on the duskside. This is also true for the pattern 2 vortices found in the reexamination cases. By contrast, Taylor et al. (2012) found vortices with a dawn-dusk asymmetry, with $71 \%$ on the dusk flank suggesting preferential growth in this region.

There may be ionospheric signatures corresponding to the magnetospheric vortices. We would expect to observe the ionospheric signatures at the footprints of the magnetic field lines that pass through the center of the magnetospheric vortices. We mapped an elliptical-shaped set of points in one of the longest lasting dayside vortices found in case 1 (and shown in Fig. 13) to a small area in the early afternoon $(\sim$ 12:00-18:00 LT) northern and southern high-latitude $\left(75^{\circ}\right)$ ionosphere, as indicated by the white crosses in Fig. 14. The solid black line in the figure marks the boundary of the open magnetic field line region. The ellipse in the magnetosphere maps to a small vortex at the convection reversal boundary in the Northern Hemisphere as indicated by the flow arrows at the circle marked by the white crosses. No corresponding vortex is seen in the Southern Hemisphere.

\subsection{Duration}

For our current study vortices for both cases had long periods of duration. However, pattern 2 vortices had substantially longer periods of duration than pattern 1 vortices, particularly on the dayside. These pattern 2 vortices lasted for hours (as shown in Figs. 2 and 6). Pattern 1 vortices for the previous study (not shown) lasted minutes before they convected tailward and started to dissipate. Those found in the re-examination (pattern 2) lasted just seconds with some of them surviving up to a few minutes. This is shown in Figs. 15 and 16 . The vortices are separated in the same four quadrants as we did for case 1 and case 2 of the fixed cases, but now for the two cases of magnetic field magnitude. Most of the vortices only lasted only a minute until they completely disappeared. Those that lasted more than a few minutes were located in the nightside region. They are not as stabilized as those formed with rotation axes on the $z$ direction for the dynamic cases, which lasted several minutes before starting to dissipate.

In the current study, most vortices did not convect, instead they rotate with axes parallel (or antiparallel) to the magnetic field. This could be the cause of slower convection vortices with longer periods of duration. For case 1 of the 18 total cases, 12 are rotating in the $x$ or $y$ direction. Of those 12,11 are located off the equator at $z> \pm 2.5$. For case 2 , we have a total of 15 cases; 12 of them are rotating in the $x$ or $y$ direction and 10 of those 12 are located at $z> \pm 2.5$. In Fig. 17 we plotted the magnetic field lines near the rotation axis of the vortex shown in Fig. 5 using the Space Weather Explorer tool from the Community Coordinated Modeling Center at NASA Goddard Space Flight Center. The magnetic field lines are shown in red and the plasma flow is shown with yellow lines. The vortex rotation axis is parallel to the magnetic field lines. The color background represents the plasma density.

\subsection{Other characteristics}

Other characteristics for the fixed conditions cases vortices are that they appear to have symmetry, and they would develop in pairs at approximately the same $x-y$ location, but with a different $z$ coordinate. Some vortices would develop simultaneously on the dawn and dusk side for the same time frame. This is shown in Fig. 8 where two vortices with almost the same size, rotation velocity and rotation axis developed at the dawn and dusk side. However, the dawnside vortex rotates counterclockwise, and the duskside vortex rotates clockwise. Figure 7 shows an example of two duskside 


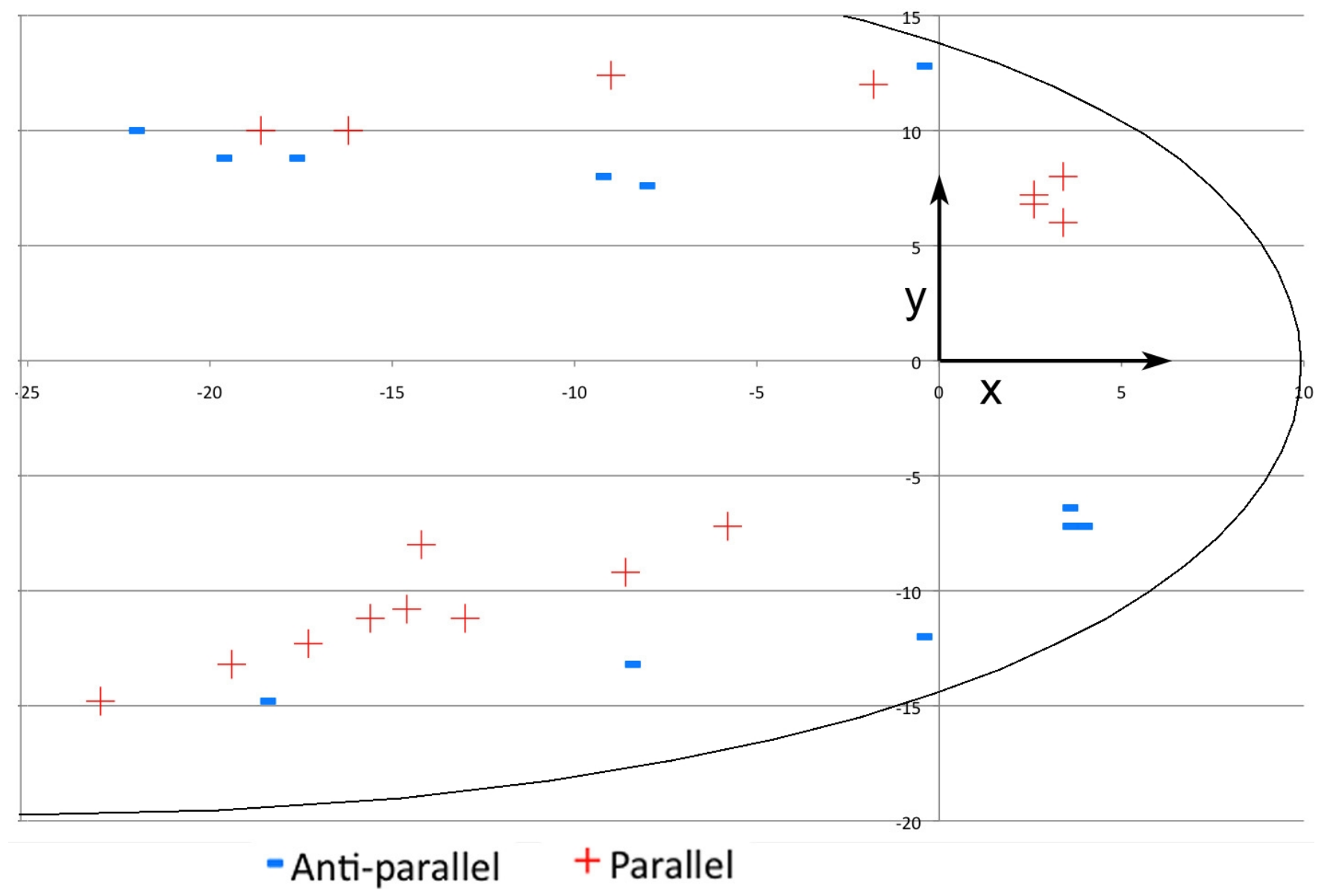

Fig. 11. $X-Y$ plot of the vortex rotation directions referred from the right-hand rule following the local magnetic field dominant component. The ones on the dayside followed a pattern; the ones on the duskside rotated parallel to the field while the ones on the dawnside rotated antiparallel. The black curve represents the magnetopause location as calculated by the BATS-R-US model for the last time step in case 2 .

vortices that formed almost with the same $x-y$ coordinates, but with totally different $z$ coordinates.

For the dynamic solar wind conditions cases (previous study), $47 \%$ of the total development of vortices formed under low speed solar wind conditions, before the onset of the high speed stream (see Collado-Vega, et al., 2007, for reference). This is also the case with the static solar wind conditions (current study), where 18 vortices formed under low solar wind speed $\left(360 \mathrm{~km} \mathrm{~s}^{-1}\right)$ and 15 vortices formed during high solar wind speed $\left(700 \mathrm{~km} \mathrm{~s}^{-1}\right)$. However, it is evident in both studies that it is necessary to have dynamic, stronger, faster and driven solar wind conditions to create faster tailward-convecting vortices.

We attribute dayside vortices like the one shown in Fig. 18 to high-latitude reconnection during northward IMF. The simultaneous appearance of such vortices in both the Northern and Southern Hemispheres (see Figs. 8 and 7) suggest simultaneous reconnection is occurring poleward of both cusps. As suggested by, for example, Dungey (1963) and also illustrated by Reiff (1984), magnetospheric plasma flows sun- ward toward the high-latitude reconnection site, reconnects, and then flows antisunward and poleward along the magnetopause. In steady state, the sunward flows form the equatorward edge of the vortices, while the antisunward flows form the poleward edge. Flows are very weak at the center of the vortices, whose locations remain fixed for the steady-state solar wind conditions in these runs.

The magnetosheath/boundary layer flow drags the closed field lines that have recently been added to the magnetosphere antisunward to the magnetotail, where they slip azimuthally into the magnetotail and ultimately contract earthward within the center of the magnetotail. Vortices in the magnetotail with axes in the $x$ direction result from the shear between newly appended magnetic field lines folding into the magnetotail at higher latitudes and the same magnetic field lines collapsing earthward deeper within the magnetotail at lower latitudes.

In previous studies, waves and vortices were also observed on the same scale size and time duration as the ones found in this study. Couzens et al. (1985) observed waves of 

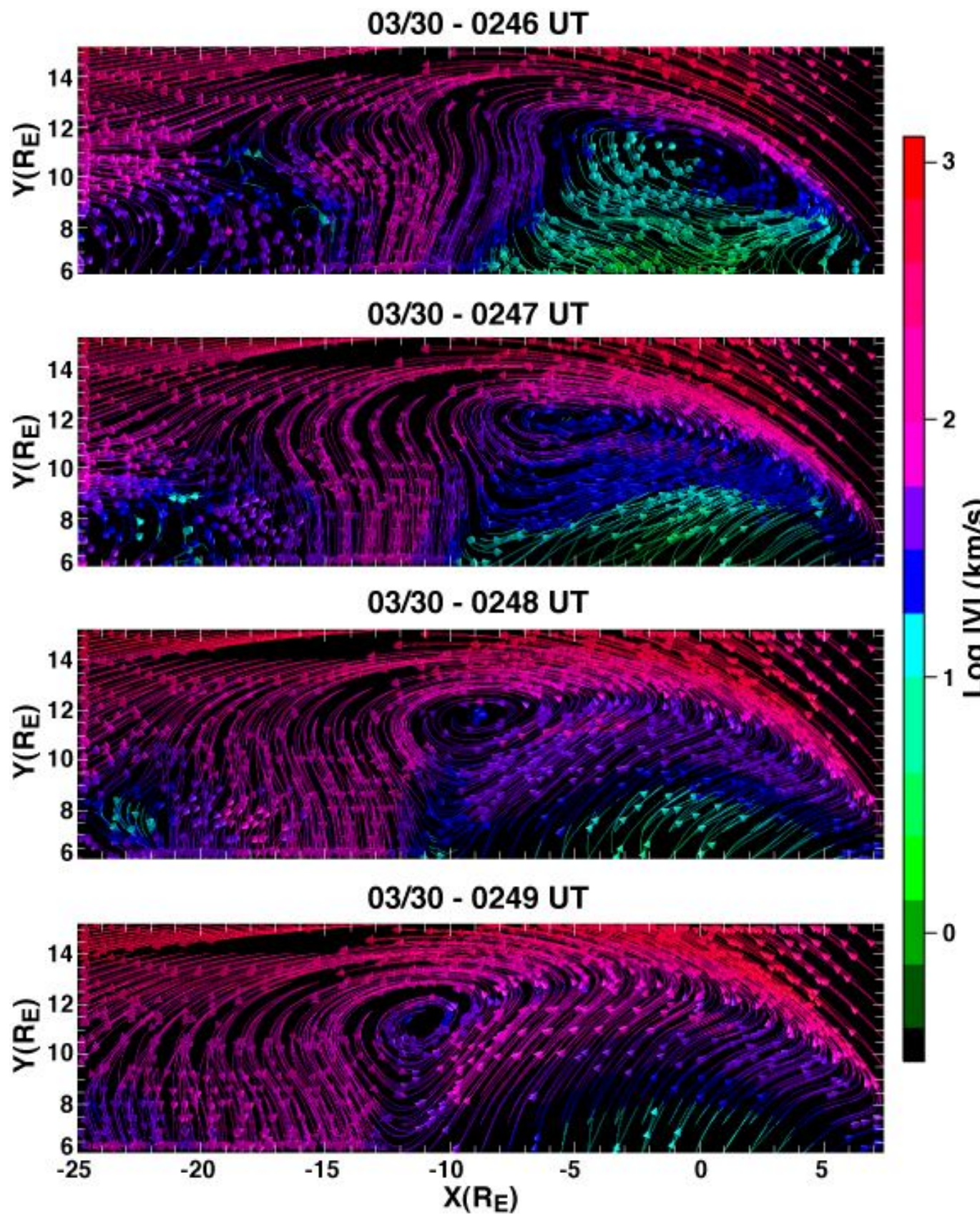

Fig. 12. Vortex progression of 30 March from $02: 46$ to $02: 49$ UT from Collado-Vega et al. (2007). Within only 4 min the vortex core travels approximately $12 R_{\mathrm{E}}$ down the flank. The velocity of rotation increases significantly along the flank, from 20 to $60 \mathrm{~km} \mathrm{~s}^{-1}$, while its traveling velocity decreases when the vortex becomes fully ordered around 02:48 UT. This is not typical of the vortices found in the present study.

Table 4. Previous observations.

\begin{tabular}{cccc}
\hline Authors & Satellite & Size & Period \\
\hline Hones et al. (1978, 1981) & ISEE 1 and 2 & $20-40 R_{\mathrm{E}}$ & Up to 9h \\
Sckope et al. (1981) & ISEE 1 and 2 & $1 R_{\mathrm{E}}$ & Few minutes \\
Couzens et al. (1985) & ISEE 1 and 2 & $11000 \mathrm{~km}$ & Several hours \\
Chen et al. (1993) & ISEE 1 and 2, IMP 8 & Wavelength 15 $R_{\mathrm{E}}$ & 5 min \\
Otto and Fairfield (2000) & Geotail & Approximately 2 $R_{\mathrm{E}}$ & $2.5 \mathrm{~min}$ \\
\hline
\end{tabular}




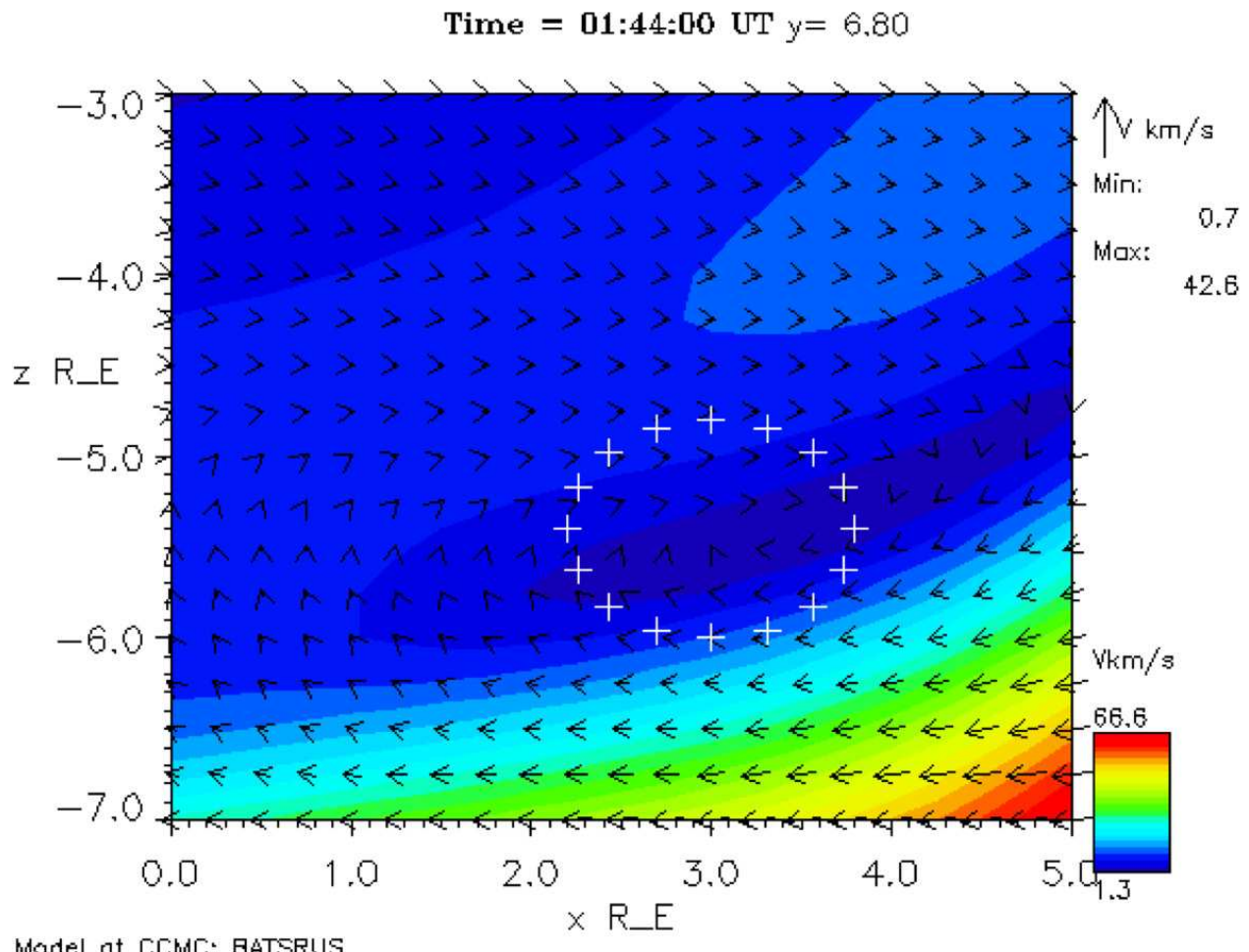

Model ot CCMC: BATSRUS

Fig. 13. Cut plane for $y=6.80 R_{\mathrm{E}}$ for a case 1 dusk dayside vortex. The color represents the velocity magnitude and the arrows the flow direction. The white marks represent the selected data points were field lines were selected to map to the ionosphere.
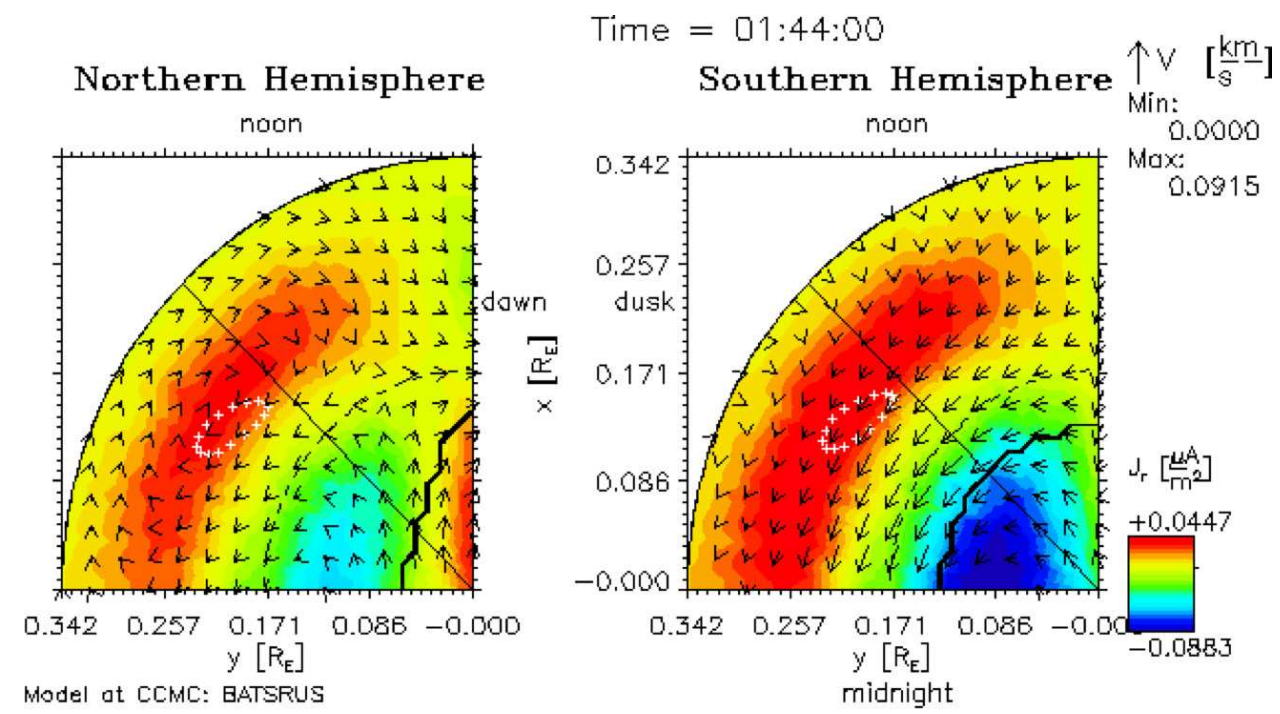

Fig. 14. Northern and Southern Hemispheres of the ionosphere at 01:44 UT. The color represents the radial component of the current and the arrows the flow direction. The solid black line marks the boundary of the region with open field lines near the magnetic poles, and the white crosses mark the location where the vortex maps into the ionosphere at about 75 degrees latitude between 12:00 and 18:00 LT. A vortex is actually present in the Northern Hemisphere ionosphere, but not in the Southern Hemisphere ionosphere.

approximately $11000 \mathrm{~km}$ that lasted for several hours using ISEE 1 and 2 data. Chen et al. (1993) observed waves with an average period of about 5 min with a wavelength of the order of $15 R_{\mathrm{E}}$ using data from ISEE 1, 2 and IMP 8 . Hones et al. $(1978,1981)$ published the first evidence for large vortices with a size of $20 R_{\mathrm{E}}$ near the plasma sheet that had periods of hours. Borovsky et al. (1997) showed $v_{x}-v_{y}$ autocorrelations in the plasma sheet that indicate vortices with periods in the 

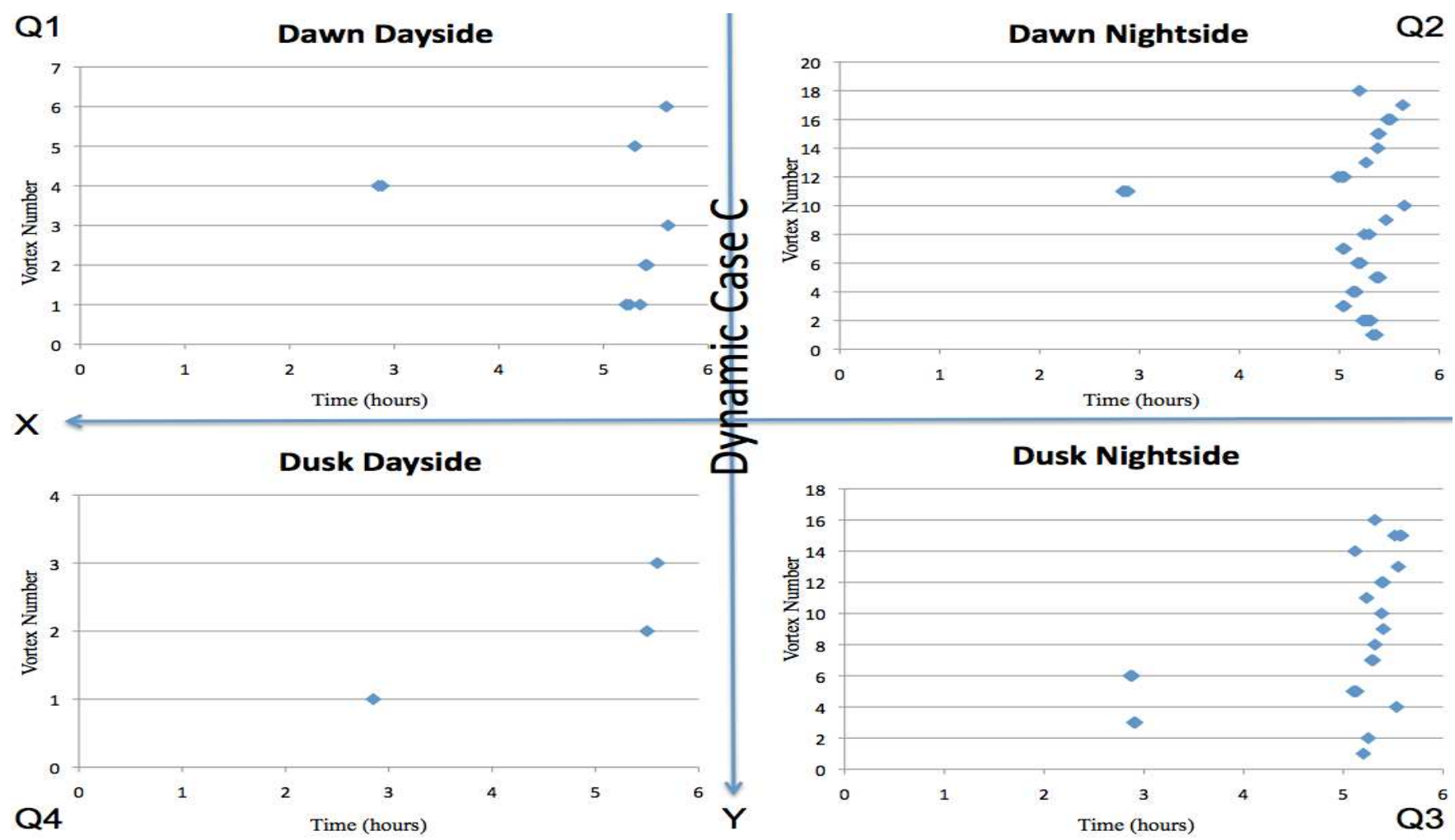

Fig. 15. Time duration of vortices for case $C$ (dynamic cases) with a 10-15 nT magnetic field magnitude. The vortices last only a few minutes before starting to dissipate. This figure only shows the duration of the vortices and not their rotation direction.

range of 6-18 min. Our events dimensions fluctuate from 2 to $6 R_{\mathrm{E}}$, and in both cases (case 1 and 2) we have vortices that have long periods of duration, some in terms of hours. A summary of previous observations similar to those found in this study are presented in Table 4.

Foullon et al. (2008) stated that the source region of $\mathrm{KH}$ waves varies in position due to the influence of the global magnetosphere geometry and that not much attention was being applied to this factor. In this study we analyzed the influence of two different solar wind speeds and a step function of the magnetic field on the magnetosphere, and we do suggest that the vortices' development depends on their local position and magnetosphere geometry. Vortices with fixed positions were found that could be the result of having steady solar wind conditions.

To be certain that the results shown in this study are not model dependent, a steady solar wind condition run with the same solar wind parameters used in case 1 above was made using the Lyon-Fedder-Mobbary global MHD code. This was the code used in the study of Collado-Vega et al. (2007). We used our automated approach and the MHD Explorer visualization tool to find vortices. Vortices exhibited the same characteristics (e.g., rotation axis and rotation direction) at similar locations to those described in this study, which used the BATS-R-US MHD code. Although the LFM code generates more vortices and these vortices convect somewhat faster than those in the BATS-R-US runs, the vortices described in this study were found using both codes, meaning that they are not an artifact of an individual code.

\section{Conclusions}

In this study, fixed solar wind conditions were simulated to examine the creation and evolution of vortices in a global MHD model for the Earth's magnetosphere. A total of 18 vortices were found for the case with nominal solar wind speed, $360 \mathrm{~km} \mathrm{~s}^{-1}$, and 15 vortices for the cases of higher solar wind speed, $700 \mathrm{~km} \mathrm{~s}^{-1}$. By contrast, Collado-Vega et al. (2007) presented statistics for a total of 304 vortices found near the ecliptic plane on the magnetopause flanks, using simulated MHD data driven by real solar wind conditions.

Foullon et al. (2010) stated that the Kelvin-Helmholtz instability was more likely to occur for wave propagation in the direction of high flow shear. We do not see more vortices for intervals of higher solar wind velocity, which may result in a higher shear on the flanks, and most of them do not move antisunward with their rotation axes in the $z$ direction. Therefore, we conclude that most of the vortices found in the current study were not caused by the KH instability. Consequently, they could be associated with transients in the solar wind-magnetosphere interaction. The largest dayside 


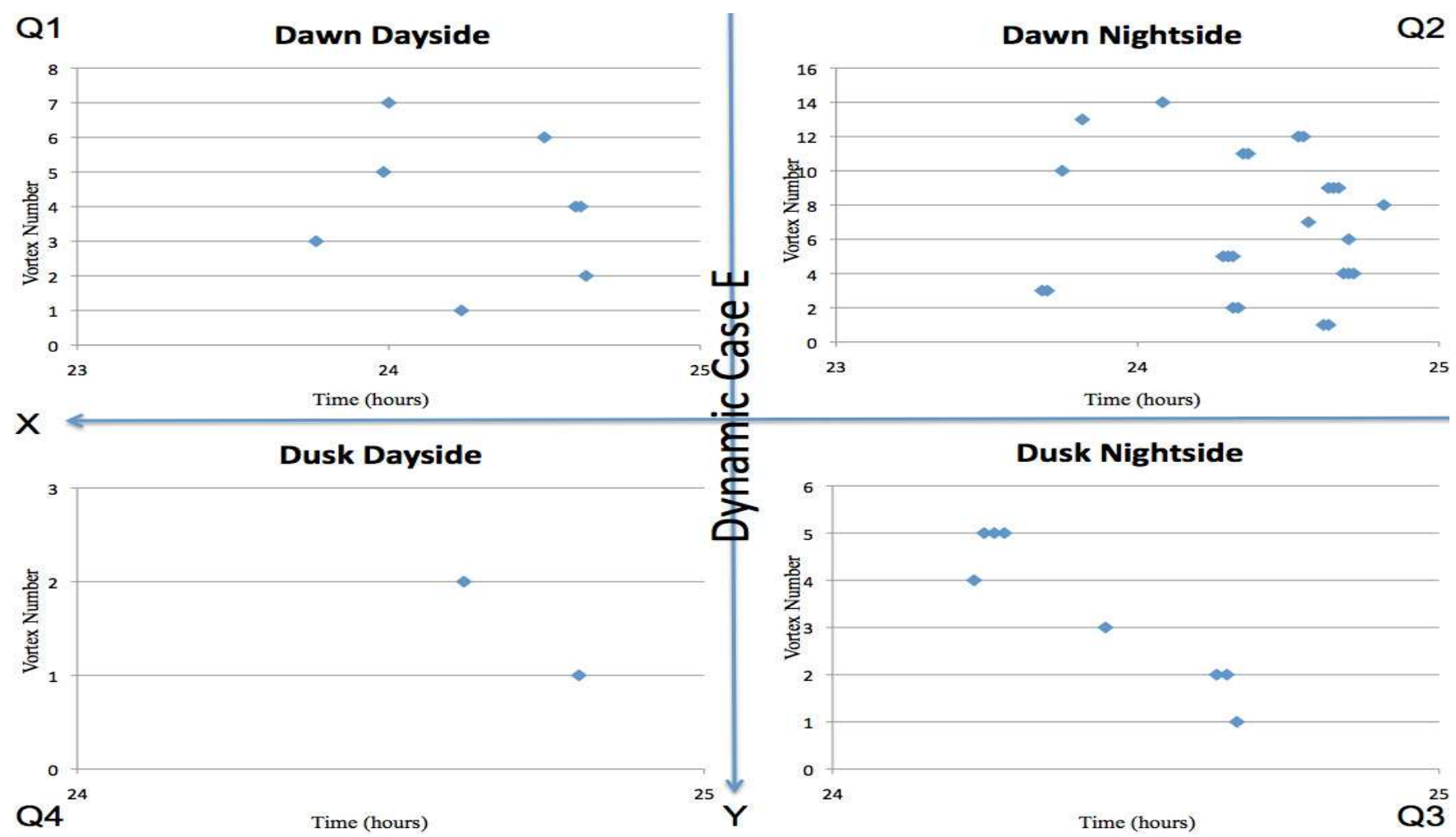

Fig. 16. Time duration of vortices for case E (dynamic cases) with a 20-25 nT magnetic field magnitude. In this case the vortices last between 2 and $5 \mathrm{~min}$ before starting to dissipate. The figure only shows their time duration.
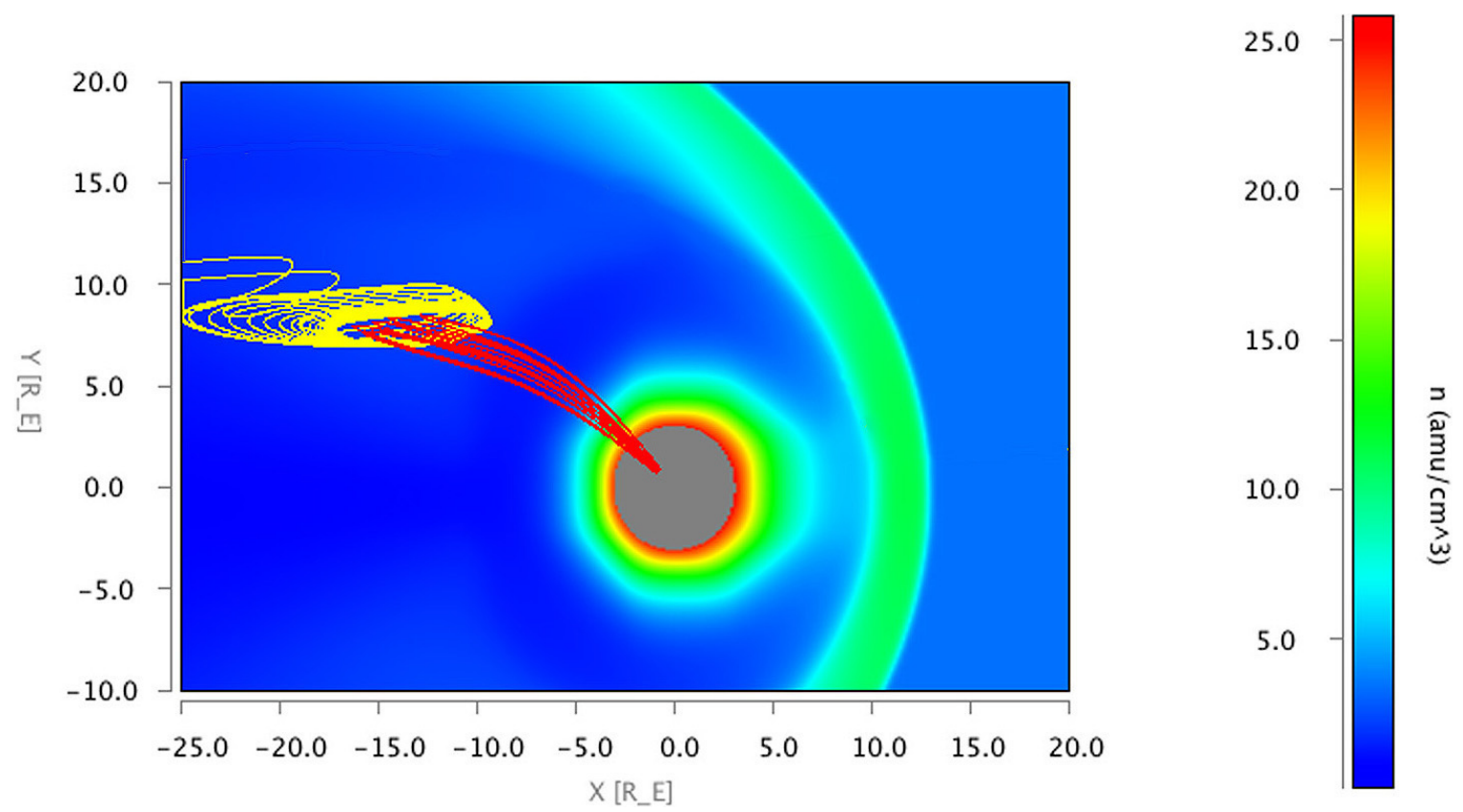

Fig. 17. A 3-D overview of the vortex in Fig. 5 using the Space Weather Explorer tool. The vortex rotation axis is parallel to the magnetic field lines. The background color represents the density, the yellow lines represent the flow and the red lines are the magnetospheric field lines. 

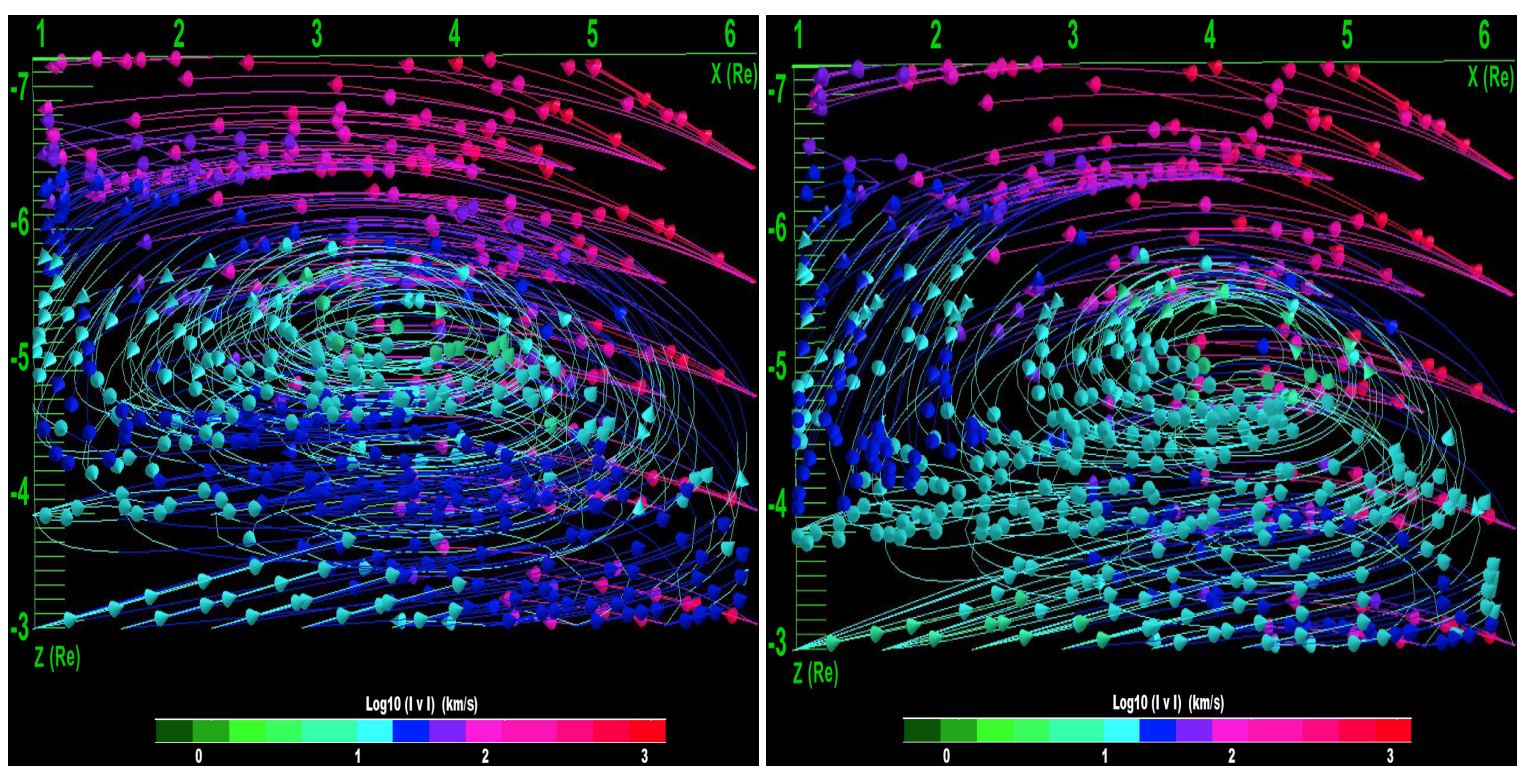

Fig. 18. Vortex developed at the dawn dayside with the rotation axis closely aligned to the $y$ axis (left). The same vortex can be encountered after $1 \mathrm{~h}$ and $16 \mathrm{~min}$ of simulation (right). This vortex has nearly a fixed position for more than an hour, but it actually moves closer to the magnetopause boundary in the sunward direction.

vortices seen in this study were the result of high-latitude reconnection happening under northward IMF. This is the first time that vortices formed under high-latitude reconnection have been visualized. Some of the vortices seen in the nightside region were also formed by the reconnected magnetospheric field lines that moved along the magnetosphere sides and got pushed back to the magnetosphere in the far tail region. The vortices were formed by the shear between the transition of the antisunward flows of recently reconnected field lines and the sunward flows of magnetospheric closed field lines.

The results of a detailed intercomparison of the new results in this paper for steady solar wind conditions and the results previously obtained for time-varying solar wind conditions reveal:

- Pattern 1 ( $z$ axis) vortices for both fixed and timevarying solar wind conditions rotate preferentially clockwise on the dawnside and counterclockwise on the duskside. This pattern is consistent with expectations for vortices driven by surface waves on the magnetopause, perhaps from the KH instability. By contrast, the sense of rotation seen in pattern 2 ( $x$ and $y$ axis) vortices does not depend on location.

- Pattern 1 ( $z$ axis) vortices that form during intervals of time-varying solar wind conditions convect antisunward in the manner expected for surface waves. The majority of pattern 1 ( $z$ axis) vortices that form during intervals of steady solar wind conditions also convect antisunward, albeit more slowly. By contrast, pattern 2 ( $x$ or $y$ axis) vortices seen during intervals of steady solar wind conditions, like those shown in Figs. 18 and 19, remain in place, sometimes dissipating. Figure 19 shows a dusk flank vortex that remains fixed for about $30 \mathrm{~min}$ of the simulation before starting to dissipate. The Fig. 18 vortex, with an axis in the $y$ direction, is part of the global convection pattern.

- Vortices developed near the equatorial dawn and dusk flanks on the nightside for both cases in the present study. However, more vortices developed at the dusk nightside for case 1 (low solar wind velocity) and more vortices developed at the dawn nightside for case 2 (high solar wind velocity). The reasons for these differences remain unclear. Pattern 1 and pattern 2 vortices generated under northward IMF for the previous study were more prevalent on the dawnside than on the duskside, and were substantially less ordered on the dawnside than on the duskside.

- Vortices formed under fixed conditions had substantially longer periods of duration than vortices formed under dynamic conditions. Vortices formed under fixed conditions had periods of duration in terms of hours. Those formed under dynamic conditions formed and dissipated in terms of several minutes. This difference can be related to the vortices convection velocity for each case. For our current study, vortices rotate either parallel or antiparallel to the magnetic field.

- As for the dynamic cases where most of the vortices formed before the onset of the high speed stream, more vortices for fixed conditions formed under the nominal 


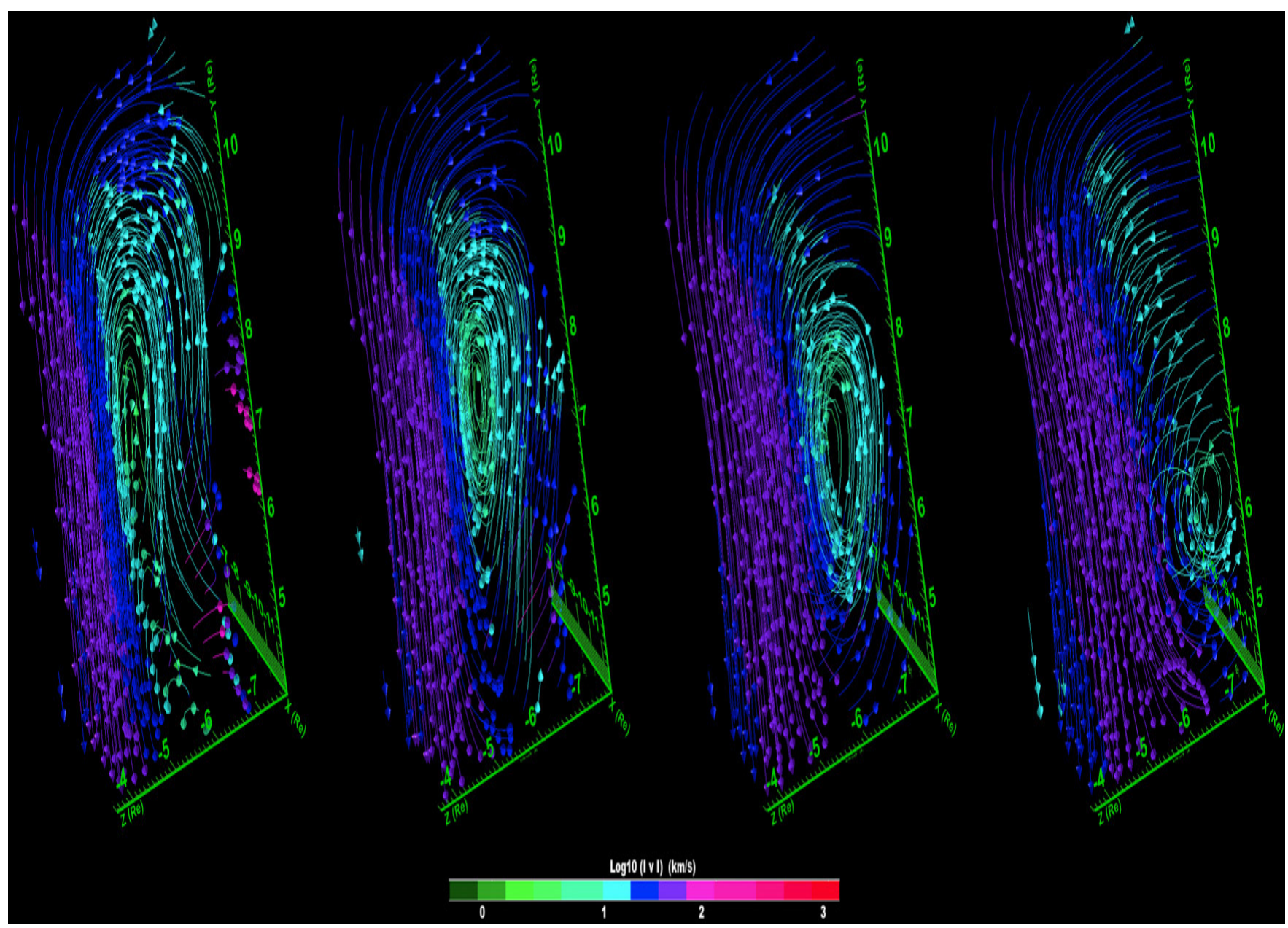

Fig. 19. This dusk flank vortex lasts for more than $30 \mathrm{~min}$. All four boxes are exactly ten minutes apart, but have the same spatial dimensions. The vortex core is not swept tailward, it remains fixed and it starts to dissipate around 02:12 UT (last box).

case of solar wind speed. For case 1 we found a total of 18 vortices, but for case 2 a total of 15 vortices were found.

- Seventy-one vortices were found rotating on the $x$ or $y$ direction for the dynamic cases (previous study) using our new search algorithm. However, these vortices have very short durations compared to the ones described in our previous study, and the two cases presented in this paper.

- We followed field lines of an elliptical-shaped set of starting points for one of the dayside vortices found in case 1, and found a vortex in the Northern Hemisphere ionosphere around 12:00-18:00 local time at 75 degrees latitude. These vortices form on closed magnetic field lines and rotate either parallel or antiparallel to the field, which suggests that their footprints couple to the ionosphere.
- The results shown in this paper are not model dependent.

Acknowledgements. Simulation results have been provided by the Community Coordinated Modeling Center at Goddard Space Flight Center through their public Runs on Request system (http://ccmc. gsfc.nasa.gov). The CCMC is a multi-agency partnership between NASA, AFMC, AFOSR, AFRL, AFWA, NOAA, NSF and ONR. The BATS-R-US Model was developed by the Center for Space Environment Modeling at the University of Michigan. The authors would like to acknowledge Tamas Gombosi as developer of the BATS-R-US code, David Berrios as developer of the Space Weather Explorer tool at the CCMC, and Jay Friedlander for his great help on the figures for this paper.

Topical Editor L. Blomberg thanks two anonymous referees for their help in evaluating this paper. 


\section{References}

Borovsky, J. E., Elphic, R., Funsten, H., and Thomsen, M.: The Earth's plasma sheet as a laboratory for flow turbulence in high- $\beta$ MHD, J. Plasma Physics, 57, 1-34, 1997.

Cai, D.-S., Li, Y., Ichikawa, T., Xiao, C., and Nishikawa, K.-I.: Visualization and criticality of magnetotail field topology in a three-dimensional particle simulation, Earth Planets Space, 53, 1011-1019, 2001.

Chen, S.-H., Kivelson, M. G., Gosling, J., Walker, R., and Lazarus, A.: Anomalous aspects of magnetosheath flow and of the shape and oscillations of the magnetopause during an interval of strongly northward interplanetary magnetic field, J. Geophys. Res., 98, 5727-5742, 1993.

Collado-Vega, Y. M., Kessel, R. L., Shao, X., and Boller, R. A.: MHD flow visualization of magnetopause boundary region vortices observed during high-speed streams, J. Geophys. Res., 112, A06213, doi:10.1029/2006JA012104, 2007.

Couzens, D. G., Parks, K., Anderson, A., Lin, R., and Reme, H.: ISEE particle observations of surface waves at the magnetosphere boundary layer, J. Geophys. Res., 90, 6343-6353, 1985.

Dungey, J. W.: Interplanetary magnetic fields and the auroral zones, Phys. Rev. Lett., 6, 47-48, 1961.

Dungey, J. W.: The structure of the exosphere or Adventures in velocity space, in: The Earth's Environment, edited by: DeWitt, C., Hieblot, J., and Lebeau, A., pp. 505-550, Gordon and Breach, New York, 1963.

Fairfield, D. H., Kuznetsova, M. M., Nagai, T., Gombosi, T. I., and Ridley, A. J.: Waves on the dusk flank boundary layer during very northward interplanetary magnetic field conditions: Observations and simulation, J. Geophys. Res., 112, A08206, doi:10.1029/2006JA012052, 2007.

Fedder, J. A., Lyon, J. G., Slinker, S. P., and Mobbary, C. M.: Topological structure of the magnetotail as function of interplanetary magnetic field and with magnetic shear, J. Geophys. Res., 100, 3613-3621, 1995a.

Fedder, J. A., Slinker, S. P., Lyon, J. G., and Elphinstone, R. D.: Global numerical simulation of the growth phase and the expansion onset for substorm observed by Viking, J. Geophys. Res., 100, 19083-19093, 1995b.

Foullon, C., Farrugia, C. J., Fazakerley, A. N., Owen, C. J., Gratton, F. T., and Torbert, R. B.: Evolution of Kelvin-Helmholtz activity on the dusk flank magnetopause, J. Geophys. Res., 113, A11203, doi:10.1029/2008JA013175, 2008.

Foullon, C., Farrugia, C. J., Owen, C. J., Fazakerley, A. N., and Gratton, F. T.: Kelvin-Helmholtz multi-spacecraft studies at the Earth's magnetopause boundaries, Twelfth International Solar Wind Conference, American Institute of Physics, 2010.

Gombosi, T. I., Powell, K. G., De Zeeuw, D. L., Clauer, C. R., Hansen, K. C., Manchester, W. B., Ridley, A. J., Roussev, I. I., Sokolov, I. V., Stout, Q. F., and Toth, G.: Solution-adaptive magnetohydrodynamics for space plasmas: Sun-to-Earth simulations, Computing in Science and Engineering, 6, 14-35, 2004.

Hasegawa, H., Fujimoto, M., Phan, T.-D., Rème, H., Balogh, A., Dunlop, M. W., Hashimoto, C., and TanDokoro, R.: Transport of solar wind into the Earth's magnetosphere through rolled-up Kelvin-Helmholtz vortices, Nature, 430, 755-758, 2004.

Hasegawa, H., Fujimoto, M., Phan, T.-D., Rème, H., Balogh, A., Dunlop, M. W., Hashimoto, C., and TanDokoro, R.: Cluster detection of rolled-up Kelvin-Helmholtz vortices at the magne- topause: comparison with simulations, Proceedings of ISSS-7, 26-31, 2005.

Hasegawa, H., Fujimoto, M., Takagi, K., Saito, Y., Mukai, T., and Rème, H.: Single-spacecraft detection of rolled-up KelvinHelmholtz vortices at the flank magnetopause, J. Geophys. Res., 111, A09203, doi:10.1029/2006JA011728, 2006.

Hones Jr., E. W., Paschmann, G., Bame, S. J., Asbridge, J. R., Sckopke, N., and Schindler, K.: Vortices in magnetospheric plasma flow, Geophys. Res. Lett., 5, 1059-1062, 1978.

Hones Jr., E. W., Birn, J., Bame, S. J., Asbridge, J. R., Paschmann, G., Sckopke, N., and Haerendel, G.: Further determination of the characteristics of magnetospheric plasma vortices with ISEE 1 and 2, J. Geophys. Res., 86, 814-820, 1981.

Hunt, J. C. R., Wray, A. A., and Moin, P.: Eddies, stream, and convergence zones in turbulent flows, Center for Turbulence Research Report CTR-S88, p. 193, 1988.

Jeong, J. and Hussain, F.: On the identification of a vortex, J. Fluid Mech., 285, 69-94, 1995.

Kessel, R. L., Chen, S.-H., Green, J. L., Fung, S. F., Boardsen, S. A., Tan, L. C., Eastman, T. E., Craven, J. D., and Frank, L. A.: Evidence of high-latitude reconnecting during northward IMF: Hawkeye observations, Geophys. Res. Lett., 23, 583-586, doi:10.1029/95GL03083, 1996.

Kivelson, M. G. and Chen, S.-H.: The Magnetopause: Surface waves and instabilities and their possible dynamical consequences, in: Physics of the Magnetopause, Geophys. Monogr. Ser., vol. 90, edited by: Song, P., Sonnerup, B. U. O., and Thomsen, M. F., pp. 257-268, AGU, Washington, D.C., 1995.

Lugt, H. J.: Vortex Flow in Nature and Technology, Krieger Publishing Company, 1995.

Lyon, J. G., Fedder, J. A., and Mobarry, C. M.: The Lyon-FedderMobarry (LMF) Global MHD Magnetospheric Simulation Code, J. Atmos. Solar-Terr. Phys., 66, 1333-1350, 2004.

Miura, A.: Dependance of the magnetopause Kelvin-Helmholtz instability on the orientation of the magnetosheath magnetic field, Geophys. Res. Lett., 22, 2993-2996, 1995.

Ogilvie, K. W. and Fitzenreiter, R. J.: The Kelvin-Helmholtz instability at the magnetopause and inner boundary layer surface, J. Geophys. Res., 94, 15113-15123, 1989.

Otto, A. and Fairfield, D. H.: Kelvin-Helmholtz instability at the magnetotail boundary: MHD simulation and comparison with Geotail observations, J. Geophys. Res., 105, 21175-21190, 2000.

Palermo, F., Califano, F., Le Contel, O., and Pegoraro, F.: Possible magnetospheric Kelvin-Helmholtz vortex signatures near the post-noon magnetopause, Memorie della Societe Astronomica Italiana Supplement, 14, p. 189, 2010.

Perry, A. E. and Chong, M. S.: A Description of Eddying Motions and Flow Patterns Using Critical-Point Concepts, Annu. Rev. Fluid Mech., 19, 125-155, 1987.

Perry, A. E. and Chong, M. S.: Topology of Flow Patterns in Vortex Motions and Turbulence, Appl. Sci. Res., 53, 357-374, 1994.

Powell, K., Roe, P., Linde, T., Gombosi, T., and De Zeeuw, D. L.: A solution-adaptive upwind scheme for ideal magnetohydrodynamics, J. Comp. Phys., 154, 284-309, 1999.

Reiff, P. H.: Evidence of magnetic merging form low-altitude spacecraft and ground-based experiments, Magnetic Reconnection in Space and Laboratory Plasmas, Geophys. Monogr. Ser., vol. 30, edited by: Hones, E. W., pp. 104-113, AGU, Washington, D.C., 1984. 
Ridley, A. J., Gombosi, T. I., and DeZeeuw, D. L.: Ionospheric control of the magnetosphere: conductance, Ann. Geophys., 22, 567-584, doi:10.5194/angeo-22-567-2004, 2004.

Robinson, S. K.: Coherent motions in the turbulent boundary layer, Annu. Rev. Fluid Mech., 23, 601, 1991.

Saunders, M. A.: Possible Kelvin-Helmholtz waves driven by reconnection accelerated flows, Geophys. Res. Lett., 16, 10311034, 1989.

Sckopke, N., Paschmann, G., Haerendel, G., Sonnerup, B. U. O., Bame, S. J., Forbes, T. G., Hones, E. W., and Russel, C. T.: Structure of the low-latitude boundary layer, J. Geophys. Res., 86, 2099-2110, 1981.

Shi, Q. Q., Pu, Z. Y., Zhang, H., Fu, S. Y., Xiao, C. J., Zong, Q.-G., Fritz, T. A., and Liu, Z. X.: Simulation studies of high-latitude magnetospheric boundary dynamics, Surv. Geophys., 26, 369386, doi:10.1007/s10712-005-1900-6, 2005.

Sibeck, D. G., Trivedi, N. V., Zesta, E., Decker, R. B., ,Singer, H. J., Szabo, A., Tachihara, H., and Watermann, J.: Pressure-pulse interaction with the magnetosphere and ionosphere, J. Geophys. Res., 108, A2, 1095, doi:10.1029/2002JA009675, 2002.

Sitar, R. J., Baker, J. B., Clauer, C. R., Ridley, A. J., Cumnock, J. A., Papitashvili, V. O., Spann, J., Brittnacher, M. J., and Parks, G. K.: Multi-instrument analysis of the ionospheric signatures of a hot flow anomaly occurring on July 24,1996 , J. Geophys. Res., 103, 23357-23372, doi:10.1029/98JA01916, 1998.

Song, P. and Russell, C. T.: Model of the formation of the lowlatitude boundary layer for strongly northward interplanetary magnetic field, J. Geophys. Res., 97, 1411-1420, 1992.
Song, P., DeZeeuw, D. L., Gombosi, T. I., Groth, C. P. T., and Powell, K. G.: A numerical study of solar wind-magnetosphere interaction for northward interplanetary magnetic field, J. Geophys. Res., 104, 28361-28378, doi:10.1029/1999JA900378, 1999.

Takagi, K., Hashimoto, C., Hasegawa, H., Fujimoto, M., and Tandokoro, R.: Kelvin-Helmholtz instability in a magnetotail flanklike geometry: Three-dimensional MHD simulations, J. Geophys. Res., 111 A08202, doi:10.1029/2006JA011631, 2006.

Taylor, M. G. G. T., Hasegawa, H., Lavraud, B., Phan, T., Escoubet, C. P., Dunlop, M. W., Bogdanova, Y. V., Borg, A. L., Volwerk, M., Berchem, J., Constantinescu, O. D., Eastwood, J. P., Masson, A., Laakso, H., Soucek, J., Fazakerley, A. N., Frey, H. U., Panov, E. V., Shen, C., Shi, J. K., Sibeck, D. G., Pu, Z. Y., Wang, J., and Wild, J. A.: Spatial distribution of rolled up Kelvin-Helmholtz vortices at Earth's dayside and flank magnetopause, Ann. Geophys., 30, 1025-1035, doi:10.5194/angeo-30-1025-2012, 2012.

Tóth, G., van der Holst, B., Sokolov, I. V., De Zeeuw, D. L., Gombosi, T. I., Fang, F., Manchester, W. B., Meng, X., Najib, D., Powell, K. G., Stout, Q. F., Glocer, A., Ma, Y.-J., and Opher, M.: Adaptive Numerical Algorithms in Space Weather Modeling, J. Computational Phys., 231, 870-903, 2012.

Zhang, H., Kivelson, M. G., Angelopoulos, V., Khurana, K. K., Walker, R. J., Jia, Y. D., McFadden, J., and Auster, H. U.: Flow vortices associated with flux transfer events moving along the magnetopause: Observations and an MHD simulation, J. Geophys. Res., 116, A08202, doi:10.1029/2011JA016500, 2011. 